\title{
The Egyptian Ascomycota 1: Genus Aspergillus
}

\author{
Abdel-Azeem AM ${ }^{1 *}$, Abu-Elsaoud AM ${ }^{1}$, Darwish $\mathrm{AMG}^{2}$, Balbool $\mathbf{B A}^{3}$, Abo Nouh $\mathbf{F A}^{1}$, \\ Abo Nahas $\mathrm{HH}^{4}$, Abdel-Azeem $\mathrm{MM}^{5}$, Ali NH ${ }^{1}$ and Kirk PM$^{6}$ \\ ${ }^{1}$ Botany Department, Faculty of Science, Suez Canal University, Ismailia 41522, Egypt.

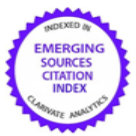 \\ ${ }^{2}$ Food Technology Department, Arid Lands Cultivation Research Institute (ALCRI), City of Scientific Research and Technological \\ Applications (SRTA-City), Alexandria, Egypt. \\ ${ }^{3}$ Microbiology Department, Faculty of Biotechnology, October University for Modern Sciences and Arts, $6^{\text {th }}$ October city, Egypt \\ ${ }^{4}$ Zoology Department, Faculty of Science, Suez Canal University, Ismailia 41522, Egypt. \\ ${ }^{5}$ Pharmacognosy Department, Faculty of Pharmacy, Suez Canal University, Ismailia 41522, Egypt. \\ ${ }^{6}$ Biodiversity Informatics \& Spatial Analysis, Royal Botanic Garden Kew, Richmond, London TW9 3AE, United Kingdom.
}

\author{
ARTICLE INFO \\ Article history \\ Accepted 30 June 2020 \\ Corresponding Editor: \\ Amrani S \\ Esmail TN \\ Vargas-de-la-Cruz C \\ Keywords \\ Biodiversity \\ Herbaria catalogues \\ Literature \\ Moubasher \\ Novel taxa \\ Taxonomy
}

Received 16 March 2020

Received revised 25 June 2020

Available online 2 July 2020

(C) Abdel-Azeem AM et al. 2020

\begin{abstract}
Since Pier Antonio Micheli described and published genus Aspergillus in Nova Plantarum Genera in 1729 the genus attracted an immense interest. The published Egyptian literature on the genus is scattered and fragmentary. By screening the available sources of information since 1921, it was possible to figure out a range of 150 taxa that could be representing genus Aspergillus in Egypt up to the present time. Ten species of Aspergillus were introduced as type materials from Egypt since 1964 till now. Recorded taxa were assigned to 5 subgenera and 25 sections. This article includes Aspergillus species that are known to Egypt, provides a comprehensive checklist of species isolated from Egypt and provisional key to the identification of reported taxa is given. Although the present study will add some new data to our information concerning the Ascomycota of Egypt, this work must be considered as a provisional one always waiting for continuous supplementation.
\end{abstract}

Published by Arab Society for Fungal Conservation

\section{Introduction}

Members of the genus Aspergillus are cosmopolitan and prevalent components of different ecosystems in a wide range of environmental and climatic zones (Klich 2002; Levic et al. 2013; Abdel-Azeem et al. 2016) because they can colonize a wide variety of substrates. Species belonging to the genus Aspergillus are widely distributed throughout the world biomes e.g. soil (Klich 2002; AbdelAzeem and Ibrahim 2004; Conley et al. 2006; Jaime-
Garcia and Cotty 2010), salt marshes (Abdel-Azeem 2003; Butinar et al. 2011; Balbool et al. 2013), agricultural ecosystems (Bayman et al. 2002; Horn 2003; Jaime-Garcia and Cotty 2006; Abdel-Azeem et al. 2007; Marín et al. 2012; Muthomi et al. 2012), arctic (Arenz et al. 2014), living biota ( $\mathrm{Yu}$ et al. 2013; Salem and Abdel-Azeem 2014; Tripathi and Joshi 2015), stones (Tang et al. 2012; Abu Deraz et al. 2016), water-related (Sivakumar et al.

\footnotetext{
* Corresponding author

E-mail address: ahmed_abdelazeem@science.suez.edu.eg (Ahmed M. Abdel-Azeem)
} 
2006; Bonugli-Santos et al. 2015), fossils (Thomas and Poinar 1988; Dörfelt and Schmidt 2005) and human (Horré et al. 2010; Marguet et al. 2012; Findley et al. 2013; Hallen-Adams and Suhr 2017).

The genus Aspergillus includes more than 340 species both pathogenic and beneficial species (Samson et al. 2014; Abdel-Azeem et al. 2016). Several species are pathogenic to plants, animals, and humans (e.g., A. fumigatus, A. terreus) and/or produce different types of toxins, such as aflatoxins and ochratoxins (e.g., A. flavus, A. ochraceous). On the other hand, several species are widely used in different industrial applications e.g. production of foods, drinks, organic acids, and a large variety of enzymes (e.g., A. niger, A. aculeatus, A. oryzae). The broad relevance and economic importance of the genus have pushed it to the forefront of fungal research, with one of the largest academic and industrial research communities dedicated to this genus (Abdel-Azeem et al. 2016).

\section{Aspergillus in Egypt}

Desert mycobiota of Egypt have been the target of many studies viz: Montasir et al. (1956a, b), Mahmoudi et al. (1964), Besada and Yusef (1968), Moubasher and Moustafa (1970), Moubasher and El-Dohlob (1970), Salama et al. (1971), Mouchacca (1971, 1973 a, b, 1977, 1982), Naguib and Mouchacca (1970), Mouchacca and Nicot (1973), Mouchacca and Joly (1974, 1976), Samson and Mouchacca (1974, 1975), Moubasher et al. (1985, 1988, 1990), Nassar (1998), Abdel-Hafez et al. (1989a, b, 1990b), Abdel-Sater (1990, 2000), Abdel-Hafez and ElMaghraby (1993), Abdel-Azeem and Ibrahim (2004) and Abdel-Azeem (1991, 2009).

Moubasher and Moustafa (1970) surveyed the Egyptian soil fungi with special reference to Aspergillus, Penicillium and Penicillium-related genera in 32 soil samples collected from the different localities in Egypt. They met sixteen species of Aspergillus and the highest population and occurrence were recorded for A. niger, A. terreus, A. flavus and A. sydowii respectively. Mouchacca and Joly (1976) studied the biodiversity of genus Aspergillus in arid soils of Egypt. They collected 31 soil samples from western desert of Egypt. They collected 14 soil samples (set A) from regions receiving very weak to null winter rains and 17 (set B) samples from regions that benefit from an appreciable amount of wintry precipitation. In their study, the taxonomic distribution is hardly affected by the dimensions of soil sand components while regional localization exerts a certain influence. Twenty-seven species of Aspergillus were isolated, some are practically omnipresent (A. niger, A. flavus group), others develop preferentially in set A soil (A. nidulans, A. ustus, A. ochraceous and possibly A. fumigatus groups) and/or have distribution positively affected (A. flavipes and A. terreus) or perhaps negatively (A. fumigatus group) due to soil reclamation.

In their extensive survey of Sinai terricolous fungi, Abdel-Azeem and Ibrahim (2004) and Abdel-Azeem (2009) recorded 17 species of Aspergillus. They recorded A. alutaceous, A. candidus, A. clavatus, A. flavus, A. fumigatus, A. japonicus, A. niger, A. ochraceous, A. sydowii, A. tamerii, A. terreus, A. ustus, A. versicolor, A. wentii, Emericella nidulans, Eurotium amstelodami and $E$. chevalieri.

Global natural hypersaline waters characterized by certain taxa mainly of Aspergillus niger and A. caesiellus, while hypersaline localities at higher environmental temperature degrees characterized by primarily or exclusively taxa of $A$. ochraceus, A. flavus, $A$. roseoglobulosus, and A. tubingensis (Abdel-Azeem et al. 2016). Aspergillus fumigatus is common in arid environments (deserts) at high temperatures, and has been found consistently in solar salterns, although it is also most abundant at salinities below 10\% $\mathrm{NaCl}$ (Moustafa 1975; El-Dohlob and Migahed 1985; Moubasher et al. 1990a; Abdel-Azeem 2003; Abdullah et al. 2010; Butinar et al. 2011; Balbool et al. 2013). The new taxa of soil representative of Emericella in Egypt were isolated also from desert saline soil as mentioned before (Samson and Mouchacca 1974, 1975).

In many parts of Egypt, several investigators studied soil fungi from cultivated soil e.g. (Abdel-Hafez 1974; Moubasher and Abdel-Hafez 1978a; Abdel-Azeem 2003). They found taxa belonging to Aspergillus, Penicillium, Fusarium, Mucor, and some dematiaceous Hyphomycetes were the most common in various types of Egyptian soils. Mazen et al. (1984) studied the fluctuation of soil fungi in wheat fields and found that the most common fungi isolated were Aspergillus represented by five species Aspergillus niger, A. terreus, A. fumigatus, A. flavus, and A. versicolor. Abdel-Hafez et al. (2000) isolated 118 species in addition to seven varieties belonging to 51 genera from cultivated and desert soils in Egypt. The results obtained from the threesoil type were basically similar, and the most common Aspergillus species were A. flavus, A. flavus var. columnaris, A. fumigatus, A. niger, Aspergillus sydowii and A. terreus.

Hafez (2012) made an ecological comparison on soil and rhizospheric fungi of maize and wheat plants in different areas in Minia Governorate in Egypt. She isolated twentyeight fungal species from wheat belonging to 18 genera and 13 species from maize belonging to 9 genera. Aspergillus was the most dominant in both rhizospheric and nonrhizospheric soils, represented by 4 species; A. niger, A. terreus, A. flavus and A. ustus. 
Fusaria and other fungi associated with rhizosphere and rhizoplane of lentil and sesame at different growth stages from cultivated soil in Egypt have been studied by AbdelHafez et al. (2012).They isolated sixteen Fusarium species and three Aspergillus species (A. flavus, A. niger and A. ochraceous).

Abdel-Azeem et al. (2007) studied the effects of longterm heavy metal contamination on diversity of terricolous fungi and nematodes in agroecosystem in Egypt as a case study. They collected 100 soil samples in a randomized way to represent different stages of land reclamation during the period from September (2004) to February (2005). These profiles represented different land use periods of 0 to 20 years. Isolated species belonged to 21 genera. The prevailing genera were Aspergillus (12 species including anamorph stages of one Emericella and one Eurotium species; 52.63\% of the total isolates). The most abundant species were: $A$. niger var. niger $(21.15 \%$ of the total isolate number), Trichoderma pseudokoningii (12.65\%), A. flavus (9.4\%) and A. fumigatus (8.63\%).

El-Morsy (1999) collected rhizospheric samples from mangrove communities and associated salt marshes plants namely: Avicennia marina, Halocnemum strobilecium, Zygophyllum album, Z. coccineum, Z. simplex, Arthrocnemum macrostachum and Limonastrum monopetalum from different places in Red Sea coast to recover their rhizosphere microfungi. He identified $A$. niveus, A. niger, A. heteromorphus, A. oryzae, A. sulphurous, A. thomii, A. terreus var. aureus, A. versicolor and Emericella nidulans.

Abdel-Moneim et al. (2010) studied the marine-derived fungi isolated from invertebrates and seaweeds of Mediterranean coast at Alexandria as a source of bioactive secondary metabolites. They recovered 8 species and one variety. They were A. flavus, A. flavus var. columinaris, $A$. fumigatus, A. japonicus, A. niger, A. ochraceus, A. tamarii, $A$. ustus and $A$. versicolor. These taxa hosted seven species of seaweeds namely; Ulva lactuca, Caulerpa racemosa and Cladophora pellucida from green alga, Jania rubens and Pterocladia capillacea from red algae and Padina pavonica and Sargassum hornschuchii from brown algae.

The mycobiota composition of the mangrove soil located in the coastal area at Red Sea in Egypt was investigated in twenty-four soil samples collected by Khalil et al. (2013). Aspergillus flavus, A. niger, A. versicolor, and A. fumigatus, recorded high species frequency in more than 15 cases out of twenty-four. AbdelAzeem et al. (2015) studied the occurrence and diversity of mycobiota in heavy metal contaminated sediments of Mediterranean coastal lagoon El-Manzala, Egypt. They found that the prevailing genera were Aspergillus (11 species including anamorph stages of two Emericella species; $36.66 \%$ of the total isolates), Penicillium (4 species 63 including anamorph of Talaromyces; 13.33\%) and the remaining taxa were represented only by two to one species each. Aspergillus niger, A. flavus and A. terreus that showed the highest percentage of frequency of occurrence.

Mustafa et al. (2013) exploited some Egyptian endophytic taxa for extracellular biosynthesis of silver nanoparticles. They isolated endophytic fungi from medicinal plants in arid Sinai. Their results showed that Zygomycota represented by two species $(9.5 \%$ of the total species number), teleomorphic Ascomycota (3 species, 14.2\%), anamorphic Ascomycota (16 species, 76.19\%). The prevailing genera were Aspergillus (3 species including anamorph stages of one Eurotium species; $14.28 \%$ of the total isolates), and Alternaria (2 species, 9.5\%). The remaining taxa were represented only by one species each. The most abundant species were: Alternaria alternata (41.6\%), Nigrospora oryzae (38.3\%) and Chaetomium globosum (11.1\%). A total 13 species belonging to 11 genera were screened for the production of AgNPs. They recorded that Aspergillus niger synthesized AgNPs in a moderate rate in comparison with other taxa.

Eight medicinal plants (Achillea fragrantissima, Artemisia herba-alba, Chiliadenus montanus, Origanum syriacum, Phlomis aurea, Tanacetum sinaicum, Teucrium polium and Thymus decussates), were screened for their content of endophytic fungi on different altitudes by Salem and Abdel-Azeem (2014) in Saint Katherine Protectorate, South Sinai, Egypt. Salem and Abdel-Azeem isolated 32 genera belonging to 75 species in which nine species of Aspergillus namely; A. alliaceus, A. bisporus, A. candidus, A. flavus, A. fumigatus, A. japonicus, A. niger, A. terreus and $A$. versicolor were recovered.

Balbool and Abdel-Azeem (2020) studied the diversity of the culturable endophytic fungi producing Lasparaginase in arid Sinai, Egypt. They recovered endophytic fungi from 23 plant species, and only four Aspergillus species; A. niger, A. flavus, A. terreus and A. nidulans, were recoverd

In Egypt, Abdel-Azeem and Rashad (2013) studied mycobiota of outdoor air that can cause asthma: a case study from Lake Manzala, Egypt. They isolated a total of 71780 mould- and 560 yeast colony-forming units from 600 exposures and the isolated taxa were assigned to 28 genera and 43 species. They found that the greater presence of fungal spores occurred in the summer. Aspergillus niger, Cladosporium cladosporioides, Epicoccum nigrum, Aureobasidium pullulans, Alternaria cheiranthi, Penicillium chrysogenum, A. fumigatus and Alternaria alternata were the predominant species. They found that Aspergillus, Cladosporium, Penicillium, and Alternaria had the greatest frequencies in air of Lake Manzala which strongly associated with allergic respiratory disease, especially asthma, in Port Said and 
Ismailia Governorates.

Kowalik and Sadurska (1973) studied the microflora (microbiota) of papyrus from samples of Cairo Museum. They recovered five species of Aspergillus; $A$. amstelodami, A. niger, A. restrictus, A. tamarii and $A$. terreus.

Mummies have been widely investigated by phenotypic and molecular techniques particularly the study of ancient bacteria and micromycetes. There are several well-known examples showing the colonization of preserved bodies by opportunistic fungi, such as the case of the restoration of the body of Ramses II, performed in Paris in 1976-77. The mummy showed a dense fungal population with species belonging to the genera Aspergillus and Penicillium (Mouchacca 1985). In his study, Mouchacca isolated 21 species and one variety of Aspergillus from debris (D) and abdominal materials (A) of Ramses II mummy. The most common species of D and A were; A. niger, A. flavus, A. versicolor, A. sydowii, A. amstelodami and A. restrictus. Aspergilli also dominated the microbial communities of the air and dust of the Egyptian mummy chamber at the Baroda Museum in India (Arya et al. 2001).

Zidan et al. (2006) studied the conservation of a wooden Graeco-Roman coffin box and they isolated Paecilomyces variotii, Penicillium aurantiogriseum, A. niger, A. flavus, A. terreus, Emericella nidulans and Mucor racemosus. These fungi were found in various parts of the coffin box, and their growth rate varied from one part to the other. Aspergillus candidus, A. ustus and A. terreus were isolated from two wooden masks dating back to the Greek- Roman period in Egypt (Darwish et al. 2013).

Abdel-Azeem et al. (2019) characterized the biodegradation that has taken place in excavated wooden objects from Abydos middle cemetery. They elucidated the type of wood degradation present, obtained information on soil properties at the site and identify fungi currently associated with the wood and soils. They recovered four species and one variety namely; Aspergillus flavus var. oryzae, A. terreus, A. niger, A. flavus and A. fumigatus from wood samples. From soil they recovered five species namely; Aspergillus flavus, A. fumigatus, A. niger, A. ochraceus and A. terreus.

Isolation and identification of mycobiota contaminants in Egyptian raw milk and various dairy products collected from different governorates were conducted by many researchers. Aspergillus sp. were recovered from Egyptian raw milk by (El-kest et al. 2015), the most common identified species were; A. flavus, A. niger, A. fumigatus, A. ochraceus, A. oryzae, A. parasiticus, A. restrictus, A. terreus and A. versicolor (Hegazy et al. 2014; Younis et al. 2016). Younis et al. (2016) isolated A. flavus, A. niger from milk powder, which was supported by Yassein et al. (2020) who reported existence of A. niger and A. terreus as 64 well.

Ras cheese (Romy) is the main traditional hard cheese in Egypt, it is manufactured in a high proportion under artisan conditions from raw milk and marketing when reaches a queried sharp flavour after 3 to 6 months.

Mycobiota of Ras cheese was extensively studied with special reference to mycotoxins production. Moubasher et al. (2018b) isolated Aspergillus sp. from Ras cheese, the most common species isolated from surface and core of examined Ras cheese samples were; A. flavus (Zommara and Rashed 2004; ELbagory et al. 2014; El-fadaly et al. 2015a, b; El-Badry and Raslan 2016; Seddek et al. 2016; Elramly et al. 2019; Ahmed et al. 2020), A. niger (ELbagory et al. 2014; El-fadaly et al. 2015a, b; El-Badry and Raslan 2016; Seddek et al. 2016; Younis et al. 2016; Elramly et al. 2019; Ahmed et al. 2020) and A. fumigatus (ELbagory et al. 2014; Seddek et al. 2016; Younis et al. 2016; Elramly et al. 2019; Ahmed et al. 2020). Other Aspergillus isolates were recovered with less frequency such as; A. alliaceus (El-fadaly et al. 2015a, b), A. candidus (Zommara and Rashed 2004; Elramly et al. 2019), A. flavipes (El-fadaly et al. 2015a, b), A. glaucus (El-fadaly et al. 2015a, b), A. nidulans (El-fadaly et al. 2015a, b; Elramly et al. 2019), A. ochraceus (El-fadaly et al. 2015a, b; El-Badry and Raslan 2016; Elramly et al. 2019; Ahmed et al. 2020), A. oryzae (El-fadaly et al. 2015a, b; Ahmed et al. 2020), A. parasiticus (Zommara and Rashed 2004; El-Badry and Raslan 2016; Younis et al. 2016; Ahmed et al. 2020), A. sydowii (Ahmed et al. 2020), A. terreus (ELbagory et al. 2014; Embaby et al. 2015; Younis et al. 2016; Elramly et al. 2019) and A. ustus (Seddek et al. 2016). Furthermore, Hegazy et al. (2014) reported isolation of A. flavus, A. fumigatus, A. japonicas, A. ochraceus, A. parasiticus and A. terreus from hard cheese. Semi-Hard cheeses are generally packed into moulds under more pressure and aged for a longer time than the soft cheeses. El-Badry and Raslan (2016) and Ahmed et al. (2020) reported the recovery of A.flavus, A. niger and $A$. ochraceus from cheddar cheese. Embaby et al. (2015) isolated A. flavus, A. niger, A. parasiticus and A. terreus from semi hard cheese (Domty), while El-kest et al. (2015) isolated Aspergillus sp. from stretched curd cheese, Mozzarella.

White cheeses with different verities compromise an important part of the Egyptian diet. Karish, Kariesh, or Kareish cheese is one of the most popular soft fresh skimmed milk, oldest cheese variety in Egypt and Arab countries (Abou-Donia 2008; Allam et al. 2017a). About $90 \%$ of the Karish cheese produced using primitive methods in the rural districts in Egypt; this traditional method affords many opportunities for microbial contamination. It is generally made from raw milk often of poor bacteriological quality, under unsatisfactory 
conditions, and sold uncovered without a container. Therefore, there is high risk of contamination and can be considered as a good medium for the growth of different types of spoilage and pathogenic microorganisms including yeasts and molds (Allam et al. 2017b). Various species of Aspergillus were reported to recover from Karish cheese samples such as; A. flavus (El-Diasty and Salem 2007; Younis et al. 2016; Moharram et al. 2018; Moubasher et al. 2018c), A. fumigatus (ELbagory et al. 2014; Hameed 2016; Moharram et al. 2018), A. niger (El-Diasty and Salem 2007; Hameed 2016; Younis et al. 2016; Moharram et al. 2018; Moubasher et al. 2018c), A. nidulans (Moharram et al. 2018) and A. terreus (ELbagory et al. 2014; Hameed 2016; Moharram et al. 2018).

White brined (pickled) cheese is a widespread cheese group produced in many varieties in hot countries such as Domiati (originated in Egypt) and Feta (originated in Greece). White brined cheese is suitable for hot climates as it is actually stored in a concentrated brine (4 to 10$\mathrm{W} \% \mathrm{NaCl}$ ) (Fox 1993). Nevertheless, Domiati cheese harbored number of fungi species including Aspergillis species such as; A. fumigatus (Hegazy et al. 2014) and $A$. niger (Hameed 2016). Egyptian Feta showed presence of Aspergillis species as well such as; A. flavus (Barakat et al. 2019; Ahmed et al. 2020), A. fumigatus, A. terreus (Hameed 2016), A. niger (Hameed 2016; Barakat et al. 2019; Ahmed et al. 2020) and A. versicolor (Hameed 2016; Barakat et al. 2019). Istanboli cheese (named after Istanbol, Turkey), is crumbly, fresh white cheese that has a delicate flavor lifted by spicy notes from being studded with Jalapeno chilies. Aspergiluus sp. isolated from Egyptian Istanboli cheese were; A. candidus, A. fumigatus, A. nidulans isolated as Emericella nidulans (Bassuony et al. 2012), A. flavus, A. nomius (Khalifa et al. 2013), A. niger and A. ochraceus (Bassuony et al. 2012; Khalifa et al. 2013). Akawi or Akawieh belongs to the group of fresh cheeses named after Akka, Palastine. It is a soft cheese made from whole goats', sheep's or cows' milk. The process used to make Akawieh is similar to that used for white cheese (Fox 1993). El-kest et al. (2015) reported isolation of Aspergillus sp. from Akawi cheese. Aspergillus was reported in other types of Egyptian white cheeses; Moharram et al. (2018) reported existence of A. carneus, A. flavus, A. fumigatus, A. niger, A. nidulans, $A$. sulphureus, A. sydowii and A. terreus in soft cheese. In white cheese; A. flavus, A. ochraceus (Hegazy et al. 2014; El-Badry and Raslan 2016), A. fumigatus, A. japonicas, A. terreus (Hegazy et al. 2014), A. niger and A. parasiticus (El-Badry and Raslan 2016), were reported. Tallaga cheese (refrigerator in Arabic), is another popular local type of packaged or unpackaged fresh soft cheeses by all socioeconomic classes in Egypt closely related to Domiati cheese and mainly ready for consumption within one 65 month of storage at refrigerator temperature (El-Kholy et al. 2016). ELbagory et al. (2014) reported recovery of $A$. flavus, A. fumigatus and A. terreus of Tallaga cheese.

Moubasher et al. (1978), reported Aspergillus sp. associated in Egyptian Roquefort cheese including; $A$. fumigatus, A. niger $A$. tamarii and $A$. versicolor. Pasteurized processed cheese products are cheese-based foods produced by comminuting, blending and melting one or more natural cheeses and optional ingredients into a smooth homogeneous blend with the aid of heat, mechanical shear and emulsifying salts (McSweeney 2007). Aspergillus sp. isolated from Egyptian processed cheese were; A. flavus (Hassanin 1993; ELbagory et al. 2014; Barakat et al. 2019; Ahmed et al. 2020), A. fumigatus (ELbagory et al. 2014; Hameed 2016; Barakat et al. 2019), A. niger (Hassanin 1993; Hameed 2016; Barakat et al. 2019; Ahmed et al. 2020), A. terreus (ELbagory et al. 2014; Hameed 2016) and A. versicolor (Barakat et al. 2019).

Yoghurt is one of the most popular dairy products worldwide, which has gained a positive perception as a healthy and natural product (El-Kholy et al. 2019). Aspergillus species isolated from yoghurt in Egypt were; A. flavus, A. niger, A. terreus (Khalifa et al. 2013; Barakat et al. 2019), A. ochraceus (Khalifa et al. 2013) and $A$. versicolor (Barakat et al. 2019). Two Aspergillus species were reported to exist in butter; A. flavus and A. niger (ElDiasty and Salem 2007; Moubasher et al. 2018c).

Dairy desserts are nutritious inexpensive dairy food prepared from milk as base constituent and cereals which considered a proper medium for fungi growth and aflatoxins production. Mahalabia, Rice Milk, Custard and Bellila are the most consumed dairy desserts in Egypt. Khalifa and Shata (2018) isolated, A. flavus, A. niger (Mahalabia, Rice Milk and Bellila), A. ochraceus (Rice milk and Mahalabia) and A. nomius (Mahalabia), while, Ahmed et al. (2020) isolated A. flavus, A. niger (Mahalabia, Custard and Rice milk) and A. terreus (Custard).

Cerelac, cornflakes and milk powder are the most consumed baby foods, so it is necessary to examine their validity. Aspergillus sp. isolated from milk powder were; A. niger (Younis et al. 2016; Yassein et al. 2020), A. terreus (Yassein et al. 2020), A. flavus (Younis et al. 2016). Yassein et al. (2020) isolated A. flavus, A. niger, A. fumigatus, A. ochraceus, A. terreus (Cerelac and cornflakes), A. nidulans isolated as Emericella nidulans (Cerelac), A. sydowii and A. versicolor (cornflakes).

Despitethe damage that Aspergillus can cause to human-being, it can also be a very promising species for many pharmaceutical leads. Some of Egyptian researchers extracted many beneficial compounds from Aspergillus metabolites e.g. quinazoline alkaloids extracted from $A$. 
nomius with antioxidant and anti-tumor activity (Ali et al. 2017). Also, A. fumigatus isolate R7 exhibited high antimicrobial activity (Shaaban et al. 2013). Awaad et al. (2017) recovered Amhezole, a novel fungal secondary metabolite, from A. terreus for treatment of microbial mouth infection. The isolated compound and the total alcoholic extract of $A$. terreus showed a remarkable activity against microbial mouth infections; Candida albicans, Lactobacillus acidophilus, Streptococcus gordonii, and S. mutan.

Aspergillus terreus dominated the biological production of the "blockbuster" drugs known as statins. The statins are a class of drugs that inhibit HMG-CoA reductase and lead to lower cholesterol production (Subhan et al. 2016).

\section{Taxonomy}

Aspergillus is traditionally classified based on morphological characteristics, such as the size and arrangement of the Aspergillary heads, the color of the conidia, the growth rate in different media and physiological characteristics.

According to these morphological characteristics, Raper and Fennell (1965) divided the genus Aspergillus into 18 groups. However, because this classification did not have any status in the nomenclature, Gams et al. (1986) introduced the use of Aspergillus subgenera and sections.

These studies showed that the groups organized by Raper and Fennell (1965), which were based on phenotypic characteristics, largely coincide with the current classifications. However, due to morphological variations in several sections resulted in controversial taxonomic groups, polyphasic identification was used, which involves the morphological, physiological, molecular and ecological characterization of a species (Samson et al. 2007). Peterson (2008) established the acceptance of five subgenera (Aspergillus, Circumdati, Fumigati, Nidulantes and Ornati) with 16 sections from a phylogenetic analysis rDNA region sequence. By contrast, (Samson and Varga 2010) based on phylogenetic analysis using multilocus sequence typing (using calmodulin, RNA polymerase 2 and the rRNA gene), subdivided Aspergillus into eight subgenera; the subgenus Aspergillus, with the sections Aspergillus and Restricti; the subgenus Fumigati, with the sections Fumigati, Clavati and Cervini; the subgenus Circumdati, with the sections Circumdati, Nigri, Flavi and Cremei; the subgenus Candidi, with the section Candidi; the subgenus Terrei, with the sections Terrei and Flavipedes; the subgenus Nidulantes, with the sections Nidulantes, Usti and Sparsi; the subgenus Warcupi, with the sections Warcupi and Zonati; and the subgenus Ornati, with the section Ornati. Later, Varga et al. (2010), based on multilocal sequence typing (using $\beta$-tubulin, calmodulin and the intergenic spacing regions [ITS] region), added the subgenus Nidulantes to the Aenei section.

Subsequently, based on different studies (Peterson 2008; Peterson et al. 2008; Varga et al. 2010; Houbraken and Samson 2011), a new classification was proposed for the genus Aspergillus that included four subgenres and 19 sections in which the subgenera Ornati and Warcupi were transferred to other genera, since they did not belong to the genus Aspergillus. Similarly, the Cremei section, which had been classified into the subgenus Aspergillus (Peterson 2008), was reclassified into the subgenus Circumdati by Houbraken and Samson (2011), resulting in the following classification; the subgenus Aspergillus, with the sections Aspergillus (teleomorph Eurotium) and Restricti (teleomorph Eurotium); the subgenus Circumdati, with the sections Candidi, Circumdati (teleomorph Neopetromyces), Flavi (Petromyces), Flavipedes (Fennellia), Nigri and Terrei; the subgenus Fumigati, with the sections Cervini, Clavati (teleomorph Neocarpenteles, Dichotomomyces) and Fumigati (Neosartorya); and the subgenus Nidulantes, with sections Aenei (teleomorph Emericella), Bispori, Cremei (teleomorph Chaetosartorya), Nidulantes (teleomorph Emericella), Ochraceorosei, Silvati, Sparsi and Usti (teleomorph Emericella). Finally, the current classification consists of six subgenera (Aspergillus, Circumdati, Cremei, Fumigati, Nidulantes and Polypaecilum) and more than 20 sections (Houbraken et al. 2014; Hubka et al. 2015; Gautier et al. 2016; Tsang et al. 2018). Recently some sections produced teleomorphic phase like Nigeri (Horn et al. 2013) and Terrei (Arabatzis and Velegraki 2013).

\section{Morphology}

Morphology forms represent an important part of the species concept of Aspergillus. Colony characters used for species characterization include; colony growth rates, texture, degree of sporulation, production of sclerotia or cleistothecia, colours of mycelia, sporulation, soluble pigments, exudates, colony reverses, sclerotia, Hülle-cells and cleistothecia. Both sexual and asexual reproduction occurs in Aspergillus and the microscopic features of these structures are important.

Diagnostic conidiophore characters include; the shape of conidial heads, the presence or absence of metulae between vesicle and phialides (i.e. uniseriate or biseriate), colour of stipes, and the dimension, shape and texture of stipes, vesicles, metulae (when present), phialides, conidia and Hülle-cells (when present). The same applies for cleistothecia, asci and ascospores. For cleistothecia (ascocarps); the development of ascomata and the way their walls are produced is also an taxonomic character. Ascospore sizes, morphology, particularly the often- 
diagnostic ornamentation (roughening, rims, wings, furrows, etc.), are considered important criteria for identifying species. Media, inoculation technique and incubation conditions affect morphological characters (Okuda 1994; Okuda et al. 2000). Samson et al. (2014) recommend the following standardized method for laboratories working on Aspergillus as shown in figure (2).

This study aims to decoument species diversity of genus Aspergillus in Egypt since 1921. Therefore, it should be mentioned here that, although the present study will add some new data to our information concerning the Ascomycota of Egypt, this updated check-list must be considered as a provisional one always waiting for continuous supplementation.

\section{Materials and Methods}

\section{Study area}

Egypt's geographical position at the junction between two large continents (Africa and Asia), and its inclusion as part of the Mediterranean basin, has indelibly influenced both the people and the biota of the country socially, economically and biologically. Egypt is a part of the Sahara of North Africa and has an area of about $1 \mathrm{M} \mathrm{km}^{2}$, divided by the River Nile into; a western part including the Libyan Desert $\left(681000 \mathrm{~km}^{2}\right)$ and an eastern part comprising the Eastern Desert $\left(223000 \mathrm{~km}^{2}\right)$, and the Sinai Peninsula $\left(61000 \mathrm{~km}^{2}\right)$. The Nile basin, comprising the valley in the south (Upper Egypt) and Nile delta in the north (Lower Egypt), forms a riparian oasis $\left(40000 \mathrm{~km}^{2}\right)$ that constitutes the densely inhabited farmlands of Egypt (Abdel-Azeem and Salem 2013).

\section{Data Collection}

The species listed here were compiled mainly from previous studies as well as information obtained from web sites, compilations, and check-lists of Egyptian fungi previously introduced by several investigators (Reichert 1921; Melchers 1931; El-Abyad and Abu-Taleb 1993; Moubasher 1993; Mouchacca 1995, 1999, 2005; El-Abyad 1997; Abdel-Azeem 2010; Moubasher et al. 2011; Moustafa and Abdel-Azeem 2011; Soliman 2016). This study extended to more than eighteen years in documenting and updating the information on Egyptian Aspergilli. A main list of Aspergillus in Egypt was developed, and the taxa are given in alphabetical order within their sections. Name corrections, authorities, and taxonomic assignments of all taxa reported, were checked against the Index Fungorum Partnership (IFP 2020) and IMI database (IMI 2020). A provisional key to the identification of reported taxa is given.

\section{Statistical analyses}

Collected data were handled and checked for normality using Kolmogorov-Smirnov and Shapiro-Wilk at 0.05 level to check whether data are parametric or nonparametric. Data were analysedanalyzed using nonparametric data analysis chi-squared and KruskalWallis at 0.05 level (Paulson 2008; Aljandali 2016).

\section{Results}

Ten species of Aspergillus were introduced as type materials from Egypt since 1964 till 2020 namely: $A$. flaschentraegeri Stolk (1964), A. egyptiacus Moubasher and Moustafa (1972) [as 'Aspergillus aegyptiacus'], A. desertorum Samson and Mouchacca (1974), A. floriformis Samson and Mouchacca (1975), A. pseudodeflectus Samson and Mouchacca (1975), A. purpureus Samson and Mouchacca (1975), A. xerophilus Samson and Mouchacca (1975), A. vadensis Samson, R.P. de Vries, Frisvad \& J. Visser (2005), A. assiutensis Moubasher and Soliman (2011) and A. gaarensis Al-Bedak and Moubasher (2020) Nom. inval., Art. 40.7 (Shenzhen). Those novel taxa were recovered from intestine of larva of Prodenia litura, desert soil, air and water.

The records are reviewed and are enumerated below in alphabetical and hierarchical order. Species of each group are given in a taxonomic sequence and accepted names are highlighted in bold. Classification of subgenus and section were followed relevant references e.g. (Klich 2002; Samson and Varga 2010; Varga et al. 2010; Samson et al. 2014; Visagie et al. 2014; Hubka et al. 2015; Frisvad et al. 2019).

A total of 150 species belonging to 25 sections of Aspergillus were recorded (Table 2, Figure 3). Six different subgenera were recognized with 25 highly significant difference in number of sections $(\mathrm{p}=0.003 * *)$.

The Nidulantes and Circumdati subgenera were represented by 10 and 8 sections as the maximum number of sections among all recognized Aspergillus subgenera. The twenty-five sections were represented by 150 significantly different species $\left(\mathrm{p}=0.015^{*}\right)$ and percentage $\left(\mathrm{p}=0.015^{*}\right)$ as revealed by Chi-squared test statistics.

Differences in numbers of species according to the sections and subgenera were non-significantinsignificant according to Kruskal-Wallis at 0.05 level. Circumdati and Nidulantes were significantly represented by highest number of species $56(37.3 \%)$ and $51(34.0 \%)$; respectively (Table 2, Figure 3). However, subgenus Ornati (problematic) was the least and represented by 1 species $(0.67 \%)$. 


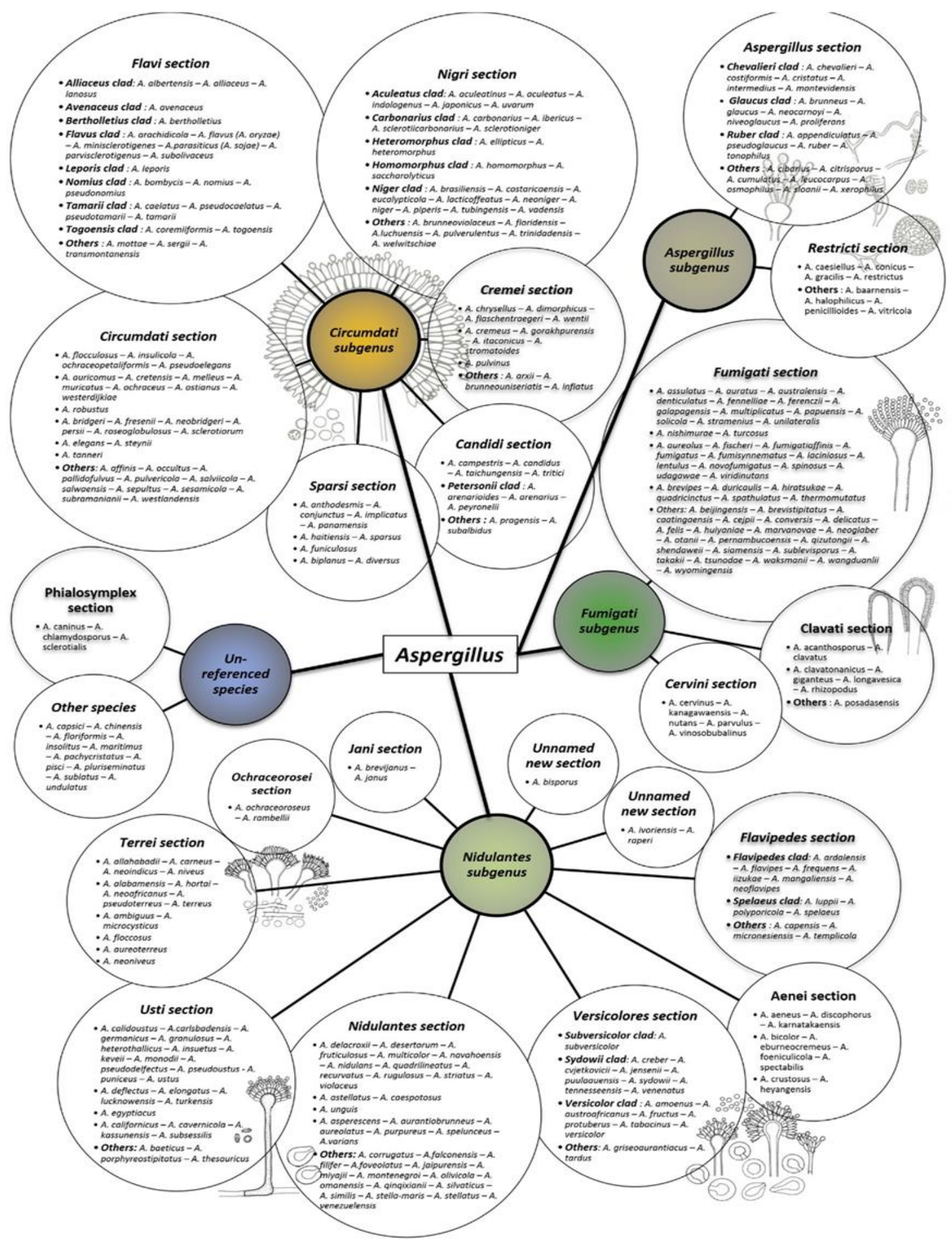

Fig 1. Classification according to their subgenus and section of 339 valid species in the Aspergillus genus (Gautier et al. 2016). 
Table 1 Overview of major subgeneric classifications of Aspergillus species (Tsang et al. 2018)

\begin{tabular}{|c|c|c|c|c|c|c|c|}
\hline Blochwitz (1929) & $\begin{array}{l}\text { Thom and Church } \\
\text { (1926) } \\
\text { Thom and Raper } \\
\text { (1945) } \\
\text { Raper and Fennell } \\
\text { (1965) }\end{array}$ & Gams et al. (1986) & (Peterson 2000) & Peterson (2008) & $\begin{array}{l}\text { Houbraken and Samson } \\
\text { (2011) }\end{array}$ & Houbraken et al.( 2014) & $\begin{array}{l}\text { Jurjević et al. (2015) } \\
\text { Kocsubé et al. (2016) } \\
\text { Sklenáŕ et al. (2017) }\end{array}$ \\
\hline $\begin{array}{l}\text { Euglobosi } \\
\text { Flavi } \\
\text { Fulvi } \\
\text { Glauci } \\
\text { Nidulantes } \\
\text { Nigroides } \\
\text { Phaei }\end{array}$ & $\begin{array}{l}\text { Group A. candidus } \\
\text { Group A. cervinus } \\
\text { Group A. clavatus } \\
\text { Group A. cremeus } \\
\text { Group A. flavipes } \\
\text { Group A. flavus } \\
\text { Group A. fumigatus } \\
\text { Group A. glaucus } \\
\text { Group A. nidulans } \\
\text { Group A. niger } \\
\text { Group A. ochraceus } \\
\text { a'Group A. ornatus } \\
\text { Group A. restrictus } \\
\text { Group A. sparsus } \\
\text { Group A. terreus } \\
\text { Group A. ustus } \\
{ }^{\mathrm{b}} \text { Group A. versicolor } \\
{ }^{\mathrm{c}} \text { Group A. wentii }\end{array}$ & 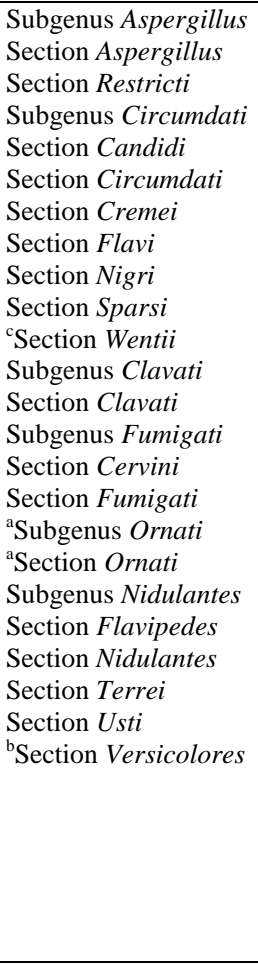 & $\begin{array}{l}\text { Subgenus Aspergillus } \\
\text { Section Aspergillus } \\
\text { Section Candidi } \\
\text { Section Cervini } \\
\text { Section Circumdati } \\
\text { Section Cremei } \\
\text { Section Flavi } \\
\text { Section Flavipedes } \\
\text { Section Nigri } \\
\text { Section Restricti } \\
\text { Section Terrei } \\
\text { Subgenus Fumigati } \\
\text { Section Clavati } \\
\text { Section Fumigati } \\
\text { Subgenus Nidulantes } \\
\text { a'Section Ornati } \\
\text { Section Nidulantes } \\
\text { Section Sparsi }\end{array}$ & 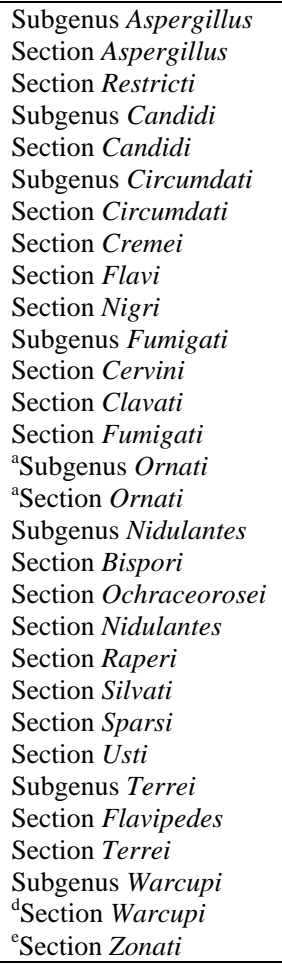 & $\begin{array}{l}\text { Subgenus Aspergillus } \\
\text { Section Aspergillus } \\
\text { Section Restricti } \\
\text { Subgenus Circumdati } \\
\text { Section Candidi } \\
\text { Section Circumdati } \\
\text { Section Flavi } \\
\text { Section Flavipedes } \\
\text { Section Nigri } \\
\text { Section Terrei } \\
\text { Subgenus Fumigati } \\
\text { Section Cervini } \\
\text { Section Clavati } \\
\text { Section Fumigati } \\
\text { Subgenus Nidulantes } \\
\text { Section Aenei } \\
\text { Section Ochraceorosei } \\
\text { Section Nidulantes } \\
\text { Section Sparsi } \\
\text { Section Usti } \\
\text { Unassigned section } \\
\text { Section Cremei }\end{array}$ & $\begin{array}{l}\text { Subgenus Aspergillus } \\
\text { Section Aspergillus } \\
\text { Section Restricti } \\
\text { Subgenus Circumdati } \\
\text { Section Candidi } \\
\text { Section Circumdati } \\
\text { Section Flavi } \\
\text { Section Flavipedes } \\
\text { Section Nigri } \\
\text { Section Terrei } \\
\text { Subgenus Fumigati } \\
\text { Section Cervini } \\
\text { Section Clavati } \\
\text { Section Fumigati } \\
\text { Subgenus Nidulantes } \\
\text { Section Aenei } \\
\text { Section Bispori } \\
\text { Section Cremei } \\
\text { Section Ochraceorosei } \\
\text { Section Nidulantes } \\
\text { Section Silvati } \\
\text { Section Sparsi } \\
\text { Section Usti }\end{array}$ & 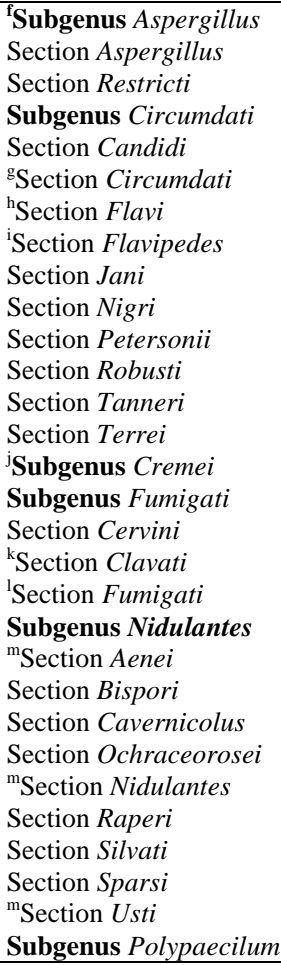 \\
\hline
\end{tabular}

\footnotetext{
${ }^{a}$ Transferred to genus Sclerocleista and excluded from Aspergillus (Subramanian 1972; Houbraken and Samson 2011)

${ }^{\mathrm{b}}$ Merged with section Nidulantes (Peterson 2000)- ${ }^{\mathrm{c}}$ Merged with section Cremei (Peterson 2008)

${ }^{\mathrm{d}}$ Transferred to genus Warcupiella and excluded from Aspergillus (Subramanian 1972; Houbraken and Samson 2011)

e Transferred to genus Warcupiella and excluded from Aspergillus (Subramanian 1972; Houbraken and Samson 2011)
Trasnferred to genus Penicilliopsis and excluded from Aspergillus (Houbraken and Samson 2011; Kocsubé et al. 2016)

${ }^{\mathrm{f}}$ Sexual synonym $=$ Eurotium $\left(\right.$ Houbraken et al. 2014) $-{ }^{\mathrm{g}}$ Sexual synonym $=$ Neopetromyces $($ Houbraken et al. 2014$)$

${ }^{\mathrm{h}}$ Sexual synonym $=$ Petromyces $\left(\right.$ Houbraken et al. 2014) $-{ }^{\mathrm{i}}$ Sexual synonym $=$ Fennellia $($ Houbraken et al. 2014$)$

${ }^{\mathrm{j}}$ Sexual synonym $=$ Chaetosartorya $\left(\right.$ Houbraken et al. 2014) $-{ }^{\mathrm{k}}$ Sexual synonym $=$ Dichotomomyces and Neocarpenteles $($ Houbraken et al. 2014)

${ }^{i}$ Sexual synonym $=$ Neosartorya $\left(\right.$ Houbraken et al. 2014) $-{ }^{\mathrm{m}}$ Sexual synonym $=$ Emericella $($ Houbraken et al. 2014$)$
} 


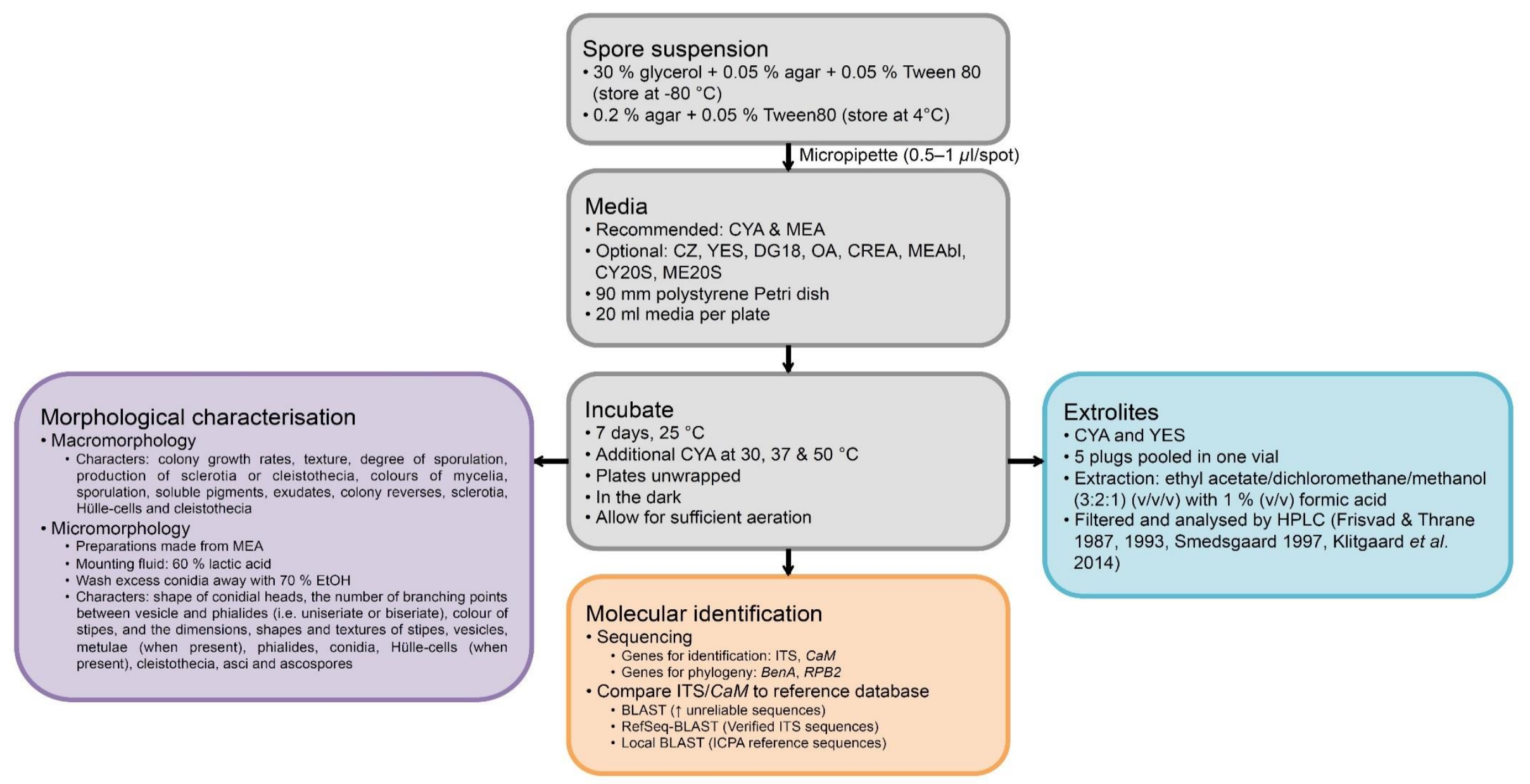

Fig 2. Flow diagram summarizing the recommended methods for the identification and characterization of Aspergillus (Frisvad and Thrane 1987, 1993; Smedsgaard 1997; Klitgaard et al. 2014) refer to methods described for detecting extrolites in fungi. 
Table 2 Subgenera and number of sections, and number of species of Aspergillus recorded in Egypt

\begin{tabular}{lccc}
\hline Subgenus & No. of sections & No. of Species & $\%$ \\
\hline Aspergillus & 2 & 22 & 14.67 \\
Circumdati & 8 & 56 & 37.33 \\
Cremei & 1 & 7.0 & 4.67 \\
Fumigati & 3 & 13 & 8.67 \\
Nidulantes & 10 & 51 & 34.00 \\
Ornati (Problematic) & 1 & 1.0 & 0.67 \\
\hline Total & 25 & 150 & 100 \\
\hline Chi-squared & $0.003^{* *}$ & $0.015^{*}$ & $0.015^{*}$ \\
\hline Kruskal-Wallis test statistic & \multicolumn{3}{|c}{4.14} \\
\hline Sign. (2-sided) df=5 & \multicolumn{3}{c}{$0.530 \mathrm{~ns}$} \\
\hline
\end{tabular}

* Significant at $p<0.05 ; * *$ Highly significant at $p<0.01 ; \mathrm{ns} ;$ Non-significant at $p>0.05$

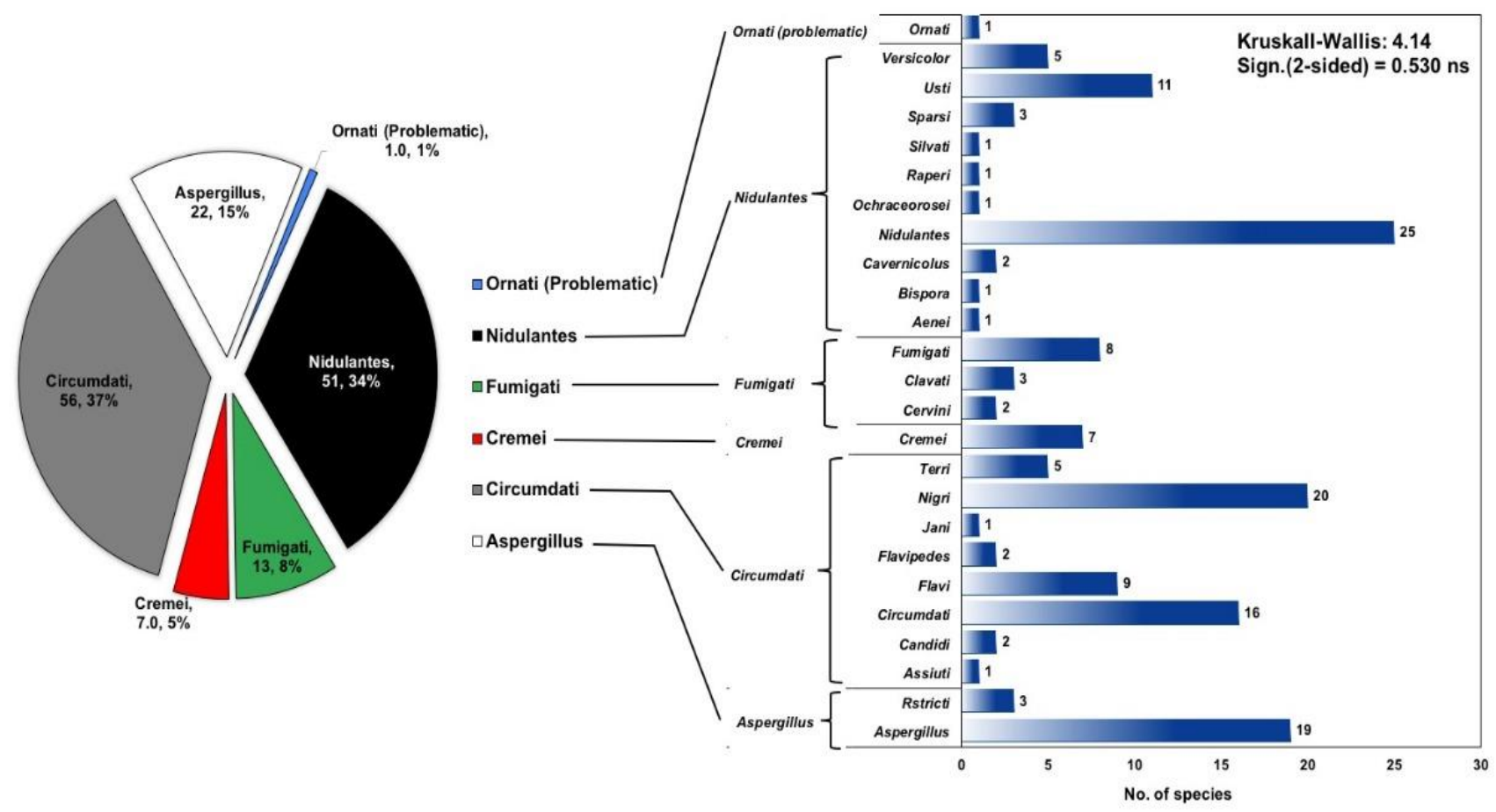

Fig 3. Distribution of different subgenera and sections. Differences between subgenera were assessed by KruskalWallis test statistic at 0.05 level. 


\section{List of Aspergillus species recorded since 1921}

Subgenus 1: Aspergillus

\section{Section 1: Aspergillus}

Aspergillus amstelodami (L. Mangin) Thom \& Church, The Genus Aspergillus: 113 (1926)

Isolated by Moubasher (1965) (IMI 114290), Abdel-Hafez et al. (1977) from salt marshes soil, and isolated as Eurotium amstelodami L. Mangin, by (Moubasher and Moustafa (1974) from air.

Aspergillus athecius Raper \& Fennell, The Genus Aspergillus: 183 (1965)

Isolated by (Moubasher et al. 2018d) from flowers from edible plants and ornamental plant; as Eurotium athecium (Raper \& Fennell) Arx by (Abdel-Hafez et al. 1995a) from plant leaf surface and desert soil.

Aspergillus chevalieri (L. Mangin) Thom \& Church, The Genus Aspergillus: 111 (1926)

Isolated by Moubasher (1969) from Triticum (IMI 142810), Abdel-Hafez et al. (1977) from soil of different localities of salt marshes, isolated as Aspergillus chevalieri var. intermedius Thom \& Raper by Abdel-Azim (1973) from Oryza sativa grains (IMI 185546), and as Eurotium chevalieri L. Mangin by (Moubasher et al. (1972) from wheat and sorghum grains.

Aspergillus cristatus Raper \& Fennell, The Genus Aspergillus: 169 (1965)

Isolated by El-Zayat et al. (2008) from Hyoscyamus muticus and as Eurotium cristatum Raper \& Fennell by Abdel-Hafez and El-Said (1997) from pepper, cinnamon and rosemary.

Aspergillus echinulatus Thom \& Church, The Genus Aspergillus: 107 (1926)

Isolated by Moubasher (1966) from soil (IMI 122165).

Aspergillus glaucus (L.) Link, Mag. Gesell. naturf.

Freunde, Berlin 3(1-2): 16 (1809)

Isolated by Sabet (1939) from different soil of sandy garden, Giza and as Eurotium herbariorum (F.H. Wigg.) Link by Ezz-ElDin (1988) from salt marshes.

Aspergillus halophilicus M. Chr., Papav. \& C.R. Benj., Mycologia 51(5): 638 (1961) [1959]

Isolated as Eurotium halophilicum C.M. Chr., Papav. \& C.R. Benj. by ( Abdel-Sater 1994) from cultivated soil at Assiut University botanical garden.
Aspergillus intermedius Blaser, Sydowia 28(1-6): 41 (1976) [1975-1976]

Isolated as Eurotium intermedium Blaser by Abdel-Hafez and El-Said (1997) from cinnamon.

Aspergillus leucocarpus Hadlok \& Stolk, Antonie van Leeuwenhoek 35: 9 (1969)

Isolated by (Moubasher et al. 2018d) from flowers of edible and ornamental plants.

Aspergillus manginii Thom \& Raper [as 'mangini'], Manual of the Aspergilli: 127 (1945)

Isolated by (Moubasher et al. 2018d) from flowers of edible and ornamental plants.

Aspergillus montevidensis Talice \& J.A. Mackinnon, C. r. Seanc. Soc. Biol. 108: 1007 (1931)

Isolated by (Moubasher and Abdel-Hafez 1978b) and as Eurotium montevidense Malloch and Cain (1972) by AbdelHafez et al. (1990a, d) from air dust particles.

Aspergillus niveoglaucus Thom \& Raper [as 'niveoglaucus'], U.S. Dept. Agric. Misc. Publ. 426: 35 (1941)

Isolated by Ismail et al. (1995) from beef carcasses and the environment in a slaughterhouse as Eurotium niveoglaucum (Thom \& Raper) Malloch \& Cain.

Aspergillus proliferans G. Sm., Trans. Br. mycol.

Soc. 26(1-2): 25 (1943)

Isolated by Moharram et al. (1989) from soybean meal.

Aspergillus pseudoglaucus Blochwitz, Annls mycol. 27(3/4): 207 (1929)

Isolated by El-Rakawy (1966) from Citrus aurantifolia (IMI 121253).

Aspergillus repens (Corda) Sacc., Michelia 2(no. 8): 577 (1882)

Isolated by El-Magraby (1989) from freshly harvested plants of crops (wheat- pea- brad bean) and isolated as Eurotium repens de Bary by (Moubasher et al. 1990a) from cultivated and salt marsh soils.

Aspergillus ruber (Jos. König, Spieck. \& W. Bremer) Thom \& Church, The Genus Aspergillus: 112 (1926)

Isolated by Moubasher et al. (1972) from corn, wheat and sorghum grains and as A. sejunctus by Moubasher (1965) from soil (IMI 116435), Eurotium rubrum König by (Moubasher et al. 1990a, b) from cultivated, desert, and salt marshes soil. 
Aspergillus tonophilus Ohtsuki, Bot. Mag., Tokyo 75: 438 (1962)

Isolated by Moharram et al. (1989) from anise and fennel seeds and isolated as Eurotium tonophilum Ohtsuki by (Abdel-Hafez et al. 1995b) from sugarcane leaves.

Aspergillus umbrosus Bainier \& Sartory, Bull. Soc. mycol. Fr. 28: 267 (1912)

Isolated from Caraway \& cumin seeds (after El-Abyad 1997).

Aspergillus xerophilus Samson \& Mouch., Antonie van Leeuwenhoek 41(3): 348 (1975)

Isolated by Samson and Mouchacca (1974) as Eurotium xerophilum Samson \& Mouch. from sandy soil, Kharga Oasis, Western Desert.

\section{Section 2: Restricti}

Aspergillus restrictus G. Sm., Journal of the Textile Institute 22(2): T100 (1931)

Isolated by Kowalik and Sadurska (1973) from Cairo museum papyrus.

Aspergillus conicus Blochwitz, Annls mycol. 12(1): 38 (1914)

Isolated by (Moharram et al. 1989a) from poultry feedstuff ingredients.

Aspergillus penicillioides Speg. (1896)

Isolated by Abdel-Azim (1975) from Oryza sativa (IMI 195573).

\section{Subgenus 2: Circumdati}

\section{Section 3: Assiuti}

Aspergillus assiutensis Moub. \& Soliman, Journal of Basic and Applied Mycology 2(1): 84 (2011)

Isolated by Moubasher and Soliman (2011) from air of grapevine plantation in ElKhawaled village, Sahel-Saleem city at approximately $25 \mathrm{Km}$ south-east of Assiut town.

\section{Section 4: Candidi}

Aspergillus campestris M. Chr., Mycologia 74(2): 212 (1982)

Isolated by Moubasher et al. (2016) from air of citrus and grapevine plantations at Assiut.

Aspergillus candidus Link, Mag. Gesell. naturf. Freunde, Berlin 3(1-2): 16 (1809)

Recorded by Reichert (1921), Melchers (1931) and isolated by Sabet (1935) from soil of sandy garden, Giza.

\section{Section 5: Circumdati}

Aspergillus alutaceus Berk. \& M.A. Curtis, in Berkeley, Grevillea 3(no. 27): 108 (1875)

Isolated by Sabet (1935) from sandy soil at Abu Sultan.

Aspergillus auricomus (Guég.) Saito, J. Ferment. Technol., Osaka 17: 3 (1939)

Isolated by Abdel-Azim (1973) from Oryza gains (IMI 176005).

Aspergillus bridgeri M. Chr., Mycologia 74(2): 210 (1982)

Isolated by Moubasher et al. (2016) from air of citrus and grapevine plantations.

Aspergillus elegans Gasperini, Morb. Limon: 328 (1887)

Isolated by Misshnicky (1964) from soil (IMI 104749).

Aspergillus flocculosus Frisvad \& Samson, in Frisvad, Frank, Houbraken, Kuijpers \& Samson, Stud. Mycol. 50(1): 33 (2004)

Isolated by Moubasher et al. (2016) from hypersaline \& alkaline lake in Wadi El Natron.

Aspergillus fresenii Subram, Hyphomycetes (New Delhi): 552 (1971)

Isolated from sandy soils and clay soil (after El-Abyad 1997).

Aspergillus gaarensis Al-Bedak \& Moubasher, Stud. Fung. 5(1): 62 (2020)

Isolated by Al-Bedak and Moubasher (2020) from a cultivated soil sample close to El-Gaar lake in Wadi-ElNatron region.

Aspergillus insulicola Montem. \& A.R. Santiago, Mycopathologia 55(2): 130 (1975)

Isolated as Moubasher et al. (2016) from soil of citrus \& grapevine plantation at Assiut.

Aspergillus melleus Yukawa, J. Coll. Agric. imp. Univ. Tokyo 3: 358 (1911)

Isolated by Samson and Mouchacca (1975) from soil sample collected in the Western Desert and isolated as Aspergillus quercinus (Bainier) Thom \& Church by Ismail et al. (2002) from air of Western Desert.

Aspergillus ochraceus G. Wilh., Inaugural Dissertation (Strassburg): 66 (1877)

Isolated by Sabet (1935) from different soil of sandy garden, Giza. 
Aspergillus ostianus Wehmer, Botan. Zbl. 80: 461 (1899)

Isolated by Moubasher et al. (2016) from air of citrus plantations

Aspergillus petrakii Vörös-Felkai Beih. Sydowia 1: 62 (1957) [1956]

Isolated by Ezz-ElDin (1988) from salt marsh soils.

Aspergillus robustus M. Chr. \& Raper, Mycologia 70(1): 200 (1978)

Isolated by Abdel-Sater et al. (2016) from citrus and grapevine plantations in the Assiut region.

Aspergillus roseoglobulosus Frisvad \& Samson, in Frisvad, Frank, Houbraken, Kuijpers \& Samson, Stud. Mycol. 50(1): 30 (2004)

Isolated by Samson and Mouchacca (1974) Moubasher et al. (2016) from hypersaline \& alkaline lake at Wadi El Natron.

Aspergillus sclerotiorum G.A. Huber, Phytopathology 23: 306 (1933)

Isolated by El-Kady et al. (1992) from soils, air, seeds, grains, food- and feed-stuff.

Aspergillus sulphureus sulphureus (Fresen.) Thom \& Church, The Aspergilli: 185 (1926)

Isolated by El-Coorani (1966) from Solanum tuberosum (IMI 116957).

\section{Section 6: Flavi}

Aspergillus alliaceus Thom \& Church, Manual of the Aspergilli: 244 (1945)

Isolated by Naguib (1966) from desert soil (IMI 121635) and as Petromyces alliaceus Malloch \& Cain by Al-Bedak (2007) from jojoba seed.

Aspergillus avenaceus G. Sm. 1943

Isolated by Shindia (1990) from composts.

Aspergillus flavofurcatus Bat. \& H. Maia, [as 'flavofurcatis'], Anais Soc. Biol. Pernambuco 13: 94 (1955)

Isolated by Abdel-Hafez et al. (1986) from air-dust particles at different Governorates.

Aspergillus flavus Link, Mag. Gesell. naturf. Freunde, Berlin 3(1-2): 16 (1809)

Isolated by Natrass (1932) from mealy bug in upper Egypt, Misshnicky (1964) from soil (IMI 104750), as A. oryzae (Ahlb.) Cohn by (Abdel-Hafez et al. 1990c) from sorghum dust at Upper Egypt, as A. oryzae var. effusus and as Petromyces flavus B.W. Horn, I. Carbone \& G.G. Moore by Hammam (2000) from wheat aphid; as A. flavus var. columnaris Raper \& Fennel by ElWakil from rhizospher (IMI 281177) and El-Maraghy (1989) from leguminous varieties and hybrids seeds, as A. variabilis Gasperini by Satour (1976) from Arachis hypogea (IMI 206096).

Aspergillus parasiticus Speare, Report Exp. Stat. Hawaiian Sugar Planters' Assoc., Path. \& Phys. Bull 12: 38 (1912)

Isolated by Natrass (1932) from mealy bug and as Petromyces parasiticus B.W. Horn, I. Carbone \& Ram.Prado by Moubasher et al. (1990) from soil.

Aspergillus subolivaceus Raper \& Fennell, The Genus Aspergillus: 385 (1965)

Isolated by Moubasher and Abdel-Hafez (1978) from desert soil, Nile valley and coastal area of Mediterranean.

Aspergillus tamarii Kita, Centbl. Bakt. ParasitKde, Abt. II 37(17/21): 432 (1913)

Isolated by Metwalty (1966) from Attacus ricini (IMI 123289) and Naguib and Mouchacca (1970) from desert soil.

Aspergillus terricola Marchal and Marchal, Revue mycol., Toulouse 15(no. 59): 101 (1893)

Isolated by Moubasher and Moustafa (1970) from soil and by Moubasher (1965) as A. terricola var. americanus Marchal \& É.J. Marchal [as 'americana'] (1921) (Thom and Church 1921) from soil (IMI 114289).

Aspergillus thomii G. Sm., (G. Sm., Trans. Br. mycol. Soc. 34(1): 17 (1951)

Isolated by El-Morsy (1999) from rhizosphere of halophytic dicots along the Red Sea coast.

\section{Section 7: Flavipedes}

Aspergillus flavipes (Bainier \& R. Sartory) Thom \& Church, Manual of the Aspergilli: 179 (1926)

Isolated by Sabet (1935) from soil of sandy garden, Giza.

Aspergillus neoflavipes Hubka, A. Nováková, M. Kolařík \& S.W. Peterson, in Hubka, Nováková, Kolarík, Varga, Jurjevic \& Peterson, Mycologia 107(1): 192 (2015) Isolated as Fennellia flavipes B.J. Wiley \& E.G. Simmons by Moubasher and Abdel-Hafez (1978) from cultivated soil. 


\section{Section 8: Jani}

Aspergillus janus Raper \& Thom, Mycologia 36(6): 556 (1944)

Isolated by Shindia (1990) from compost and by El-Morsy (1999) as A. janus var. brevis from salt marsh soil.

\section{Section 9: Nigri}

Aspergillus aculeatinus Noonim, Frisvad, Varga \& Samson, Int. J. Syst. Evol. Microbiol. 58(7): 1733 (2008) Isolated by Abdel-Sater et al. (2016) from soil of citrus and grapevine plantations

Aspergillus aculeatus Iizuka, J. agric. Chem. Soc. Japan 27: 806 (1953)

Isolated by (Abdel-Hafez et al. 1990c) from wheat \& sorghum dust, Upper Egypt.

Aspergillus awamori Nakaz., Rep. Govt Res. Inst. Dep. Agric., Formosa: 1 (1907)

Isolated by Esaily (1965) from Trifolium alexandrinum rhizosphere (IMI 112712) and as A. luchuensis Inui by Ragab (1956) from soil samples.

Aspergillus brasiliensis Varga, Frisvad \& Samson, Int. J. Syst. Evol. Microbiol. 57(8): 57 (2007)

Isolated by Abdel-Sater et al. (2016) from soil of citrus and grapevine plantations

Aspergillus carbonarius (Bainier) Thom, in Thom \& Currie, Journal of Agricultural Research 7: 12 (1916)

Isolated by El-Abyad et al. (1982) from rhizospheres of some halophytic plants.

Aspergillus costaricensis Samson \& Frisvad, [as 'costaricaensis'], in Samson, Houbraken, Kuijpers, Frank \& Frisvad, Stud. Mycol. 50(1): 52 (2004)

Isolated by Abdel-Sater et al. (2016) from soil of citrus and grapevine plantations.

Aspergillus ellipticus Raper and Fennell, The Genus Aspergillus: 319 (1965)

IMI 278384.

Aspergillus ficuum (Reichardt) Thom \& Currie, Journal of Agricultural Research 7: 12 (1916)

Isolated by Zohri et al. (2014) from foodstuffs.

Aspergillus foetidus Thom \& Raper, Manual of the

Aspergilli: 219 (1945)

Isolated by Salim (1967) (IMI 130408) and as A. foetidus var. pallidus (Nakaz, Simo \& A.Watan) Raper and Fennell
(1965) by Zohri et al. (2014) from food stuff, Sohag.

Aspergillus fonsecaeus Thom \& Raper, Manual of the Aspergilli: 227 (1945)

Isolated from clay soils (after El-Abyad 1997).

Aspergillus helicothrix Al-Musallam, Antonie van Leeuwenhoek 46(4): 407 (1980)

IMI 278383.

Aspergillus heteromorphus Bat. \& H. Maia, Anais Soc. Biol. Pernambuco 15(1): 194 (1957)

Isolated by El-Morsy (1999) from rhizosphere of

halophytic dicots along the Red Sea coast.

Aspergillus japonicus Saito, Bot. Mag., Tokyo 20: 61 (1906)

Isolated by Moubasher and Abdel-Hafez (1978) from

cultivated desert salt marshes soil.

Aspergillus lacticoffeatus Frisvad \& Samson, in Samson, Houbraken, Kuijpers, Frank \& Frisvad, Stud. Mycol. 50(1): 52 (2004)

Isolated by Moharram et al. (2013) from patients at Assiut university hospital.

Aspergillus niger Tiegh., Annls Sci. Nat., Bot., sér. 5 8: 240 (1867)

Isolated by Sabet (1935) from marsh soil; as A. phoenicis (Corda) Thom \& Currie by Reichert (1921) from soil samples; A. niger var. phoenicis by Sabet (1939); A. niger var. niger by Abdel-Azeem et al. (2007) from water samples of Manzla lake.

Aspergillus pulverulentus (Mcalpine) Thom, The Genus Aspergillus: 179 (1926)

Isolated by Moubasher et al. (2015) from soil of wadi El Natraun.

Aspergillus sclerotiicarbonarius Noonim, Frisvad, Varga \& Samson, Int. J. Syst. Evol. Microbiol. 58(7): 1733 (2008)

Isolated by Abdel-Sater et al. (2016) from soil of citrus and grapevine plantations.

Aspergillus tubingensis Mosseray, La Cellule 43: 245 (1934)

Isolated by Abdel-Sater et al. (2016) from soil of citrus and grapevine plantations.

Aspergillus vadensis Samson, R.P. de Vries, Frisvad \& J. Visser, Antonie van Leeuwenhoek 87(3): 201 (2005) in (de Vries et al. 2005) 
Reported by Samson et al. 2005 (CBS 113365) isolated from air in by A.H. Moubasher.

Aspergillus violaceofuscus Gasperini, Morb. Limon: 326 (1887)

Isolated from sandy and clay soils (after El-Abyad 1997).

\section{Section 10: Terrei}

Aspergillus aureoterreus Samson, S.W. Peterson, Frisvad \& Varga, Stud. Mycol. 69: 45 (2011)

Isolated by (Moubasher et al. 2018d) from flowers from edible plants and ornamental plant and isolated as $A$. terreus var. aureus Thom \& Raper by Abdel-Hafez et al. (1985) from air samples.

Aspergillus carneus Blochwitz, Annls mycol. 31(1/2): 81 (1933)

Isolated by Moubasher (1963) from soil (IMI 101526).

Aspergillus neoniveus Samson, S.W. Peterson, Frisvad \& Varga, Stud. Mycol. 69: 53 (2011)

Isolated as Fennellia nivea (B.J. Wiley \& E.G. Simmons) Samson (1979) by (Moubasher et al. 1990b) from cultivated, desert, salt marshes soil, isolated as A. niveus by Moubasher (1965) from soil (IMI 114278) and isolated as Emericella nivea Wiley \& Simmons by El-Said and Abdel-Hafez (1995) from air above banana fields.

Aspergillus terreus Thom, in Thom \& Church, Am. J. Bot. 5: 84 (1918)

Isolated by Sabet (1935) from soil, by Moubasher and Moustafa (1970) from soil samples as A. terreus var. africanus Fennell \& Raper (1955).

Aspergillus terreus var. terreus Thom, in Thom \& Church, Am. J. Bot. 5: 84 (1918)

Isolated from air; submerged mud; coprophilous (camel dung L); thermotolerant; clay soils and Sinai; sandy, saline clay and Red Sea soils ; mangrove soils; seeds; osmophilic \& halotolerant; isolated from sheep, rabbits, camels \& buffalos hairs, human hairs, sewage sludge, chickens claws, male $\&$ female finger $\&$ toe nails, ears, noses, teeth by hair baits (after El-Abyad 1997).

\section{Subgenus 3: Cremei}

\section{Section 11: Cremei}

Aspergillus chrysellus Kwon-Chung \& Fennel, in Raper \& Fennell, The Genus Aspergillus: 424 (1965)

Isolated by Abdel-Kareem (2010) from deteriorating historical textiles in the Egyptian Museum and the Coptic Museum in Cairo.
Aspergillus cremeus Kwon-Chung \& Fennell, in Raper \& Fennell, The Genus Aspergillus: 418 (1965)

Isolated by (Abdul-Wahid 1990) from cultivated soils.

Aspergillus dimorphicus B.S. Mehrotra \& R. Prasad, Trans. Br. mycol. Soc. 52(2): 331 (1969)

Isolated by Moubasher et al. (2016) from air citrus and grapevine plantations at Assiut.

Aspergillus flaschentraegeri Stolk, Trans. Br. mycol. Soc. 47(1): 123 (1964)

Isolated by Stolk (1964) from intestine of a Prodenia litura larva.

Aspergillus pulvinus Kwon-Chung \& Fennell, in Raper \& Fennell, The Genus Aspergillus: 455 (1965)

Isolated by Zohri et al. (2014) from foodstuffs.

Aspergillus sepultus Tuthill \& M. Chr., Mycologia 78(3): 475 (1986)

Isolated by El-Kady et al. (1992) from Egyptian soils, air, seeds, grains, food- and feed-stuff.

Aspergillus wentii Wehmer, Centbl. Bakt. ParasitKde, Abt. I 2: 150 (1896)

Isolated by Sabet (1935) from soil in Nile delta.

\section{Subgenus 4: Fumigati}

\section{Section 12: Cervini}

Aspergillus cervinus Massee, Bull. Misc. Inf., Kew (4): 158 (1914)

Isolated by Moubasher and Abdel-Hafez (1978) from soil of different localities in Egypt

Aspergillus parvulus G. Sm., Trans. Br. mycol. Soc. 44(1): 45 (1961)

Isolated by Abdel-Kader et al. (1979) from barley grains.

\section{Section 13: Clavati}

Aspergillus clavatonanicus Bat., H. Maia \& Alecrim, [as 'clavato-nanica'], Anais V Congr. Soc. bot. Brasil 15: 197 (1955)

Isolated by Moubasher and Abdel-Hafez (1978) from soil of different localities.

Aspergillus clavatus Desm., Annls Sci. Nat., Bot., sér. 2 2: 71 (1834)

Isolated by El Esaily (1965) from rhizospheric soil of Vicia faba (IMI 112754). 
Aspergillus giganteus Wehmer, Mém. Soc. Phys. Hist. nat. Genève 33(2): 85 (1901)

Isolated by Elgindy (1975) from rhizosphere soil of Zea mays (IMI 191053).

\section{Section 14: Fumigati}

Aspergillus brevipes G. Sm., Trans. Br. mycol. Soc. 35(4): 241 (1952)

Isolated by Shindia (1990) from composts.

Aspergillus duricaulis Raper and Fennell, The Genus Aspergillus: 249 (1965)

Isolated by Ammar et al. (2017) from seeds.

Aspergillus fennelliae Kwon-Chung \& S.J. Kim, Mycologia 66(4): 629 (1974)

IMI 278382.

Aspergillus fischeri Wehmer, Centbl. Bakt. ParasitKde, Abt. I 18: 390 (1907)

Isolated by Kamara (1964) from Zea mays (IMI 108386), Abdel-Hafez et al. (1977) from salt marshes and isolated as Neosartorya fischeri Wehmer var. fischeri Malloch \& Cain by Abdel-Hafez et al. (1977) from salt marsh soils; Neosartorya fischeri var. glaber Wehmer Malloch \& Cain by Abdul-Wahid (1990) from cultivated soil; Neosartorya spinosa (Raper \& Fennell) Kozak. by Krug and Khan (1999) from soil samples from New valley.

Aspergillus fumigatus Fresen., Beitr. Mykol. 3: 81 (1863)

Isolated by Moubasher (1965) from soil (IMI 114282) and isolated by Moubasher and Moustafa (1970) from soil as A. fumigatus var. fumigatus and as A. fumigatus var. albus by Moubasher (1984) from soil (IMI 287535).

Aspergillus neoellipticus Kozak., Mycol. Pap. 161: 55 (1989)

Isolated as Aspergillus fumigatus var. ellipticus Raper \& Fennell by Hamed (2016) from farmland in Al Sharqia.

Aspergillus turcosus S. B. Hong, Frisvad \& Samson, (Antonie van Leeuwenhoek 93(1-2): 97 (2008)

Isolated by Moubasher et al. (2016) from hypersaline \& alkaline lake at Wadi El Natron.

Aspergillus viridinutans Ducker \& Thrower, in McClennon, Ducker \& Thrower, Aust. J. Bot. 2(3): 357 (1954)

Isolated by Abdel-Sater et al. (2016) from soil of citrus \& grapevine at Assiut.

\section{Subgenus 5: Nidulantes}

\section{Section 15: Aenei}

Aspergillus bicolor M. Chr. \& States, in Christensen, Raper \& States, Mycologia 70(2): 337 (1978)

Isolated as Emericella bicolor by Ismail et al. (1995) from various substrates.

\section{Section 16: Bispori}

Aspergillus bisporus Kwon-Chung \& Fennell, Mycologia 63(3): 479 (1971)

Isolated by Salem and Abdel-Azeem (2014) from medicinal plants in Saint Katherine Protectorate, South Sinai.

\section{Section 17: Cavernicolus}

Aspergillus egyptiacus Moub. \& Moustafa, ([as 'aegyptiacus'], J. Bot. un. Arab Repub. 15(1): 153 (1972)

Isolated by Moubasher and Moustafa (1972) from olive tree plantation.

Aspergillus subsessilis Raper and Fennell, (The Genus Aspergillus: 530 (1965)

Isolated by Samson and Mouchacca (1974) from desert soil.

\section{Section 18: Nidulantes}

Aspergillus aurantiobrunneus Raper and Fennell, The Genus Aspergillus: 511 (1965)

Isolated as Emericella aurantiobrunnea by Ismail et al. (1995) from various substrates.

Aspergillus aureolatus Munt. -Cvetk. \& Bata, Bulletin Inst. Bot. Univ. Belgrade, N.S. 1(3): 196 (1964) [1961] Isolated by El-Kady et al. (1992) from ginger, laurel, mastic and safflower

Aspergillus caespitosus Raper \& Thom, Mycologia 36(6): 563 (1944)

Isolated by Moubasher (1966) from soil (IMI 122167).

Aspergillus desertorum (Samson \& Mouch.) Samson, Visagie \& Houbraken, n Samson, Visagie, Houbraken, Hong, Hubka, Klaassen, Perrone, Seifert, Susca, Tanney, Varga, Kocsubé, Szigeti, Yaguchi \& Frisvad, Stud. Mycol. 78: 155 (2014)

Isolated as Emericella desertorum by Samson and Mouchacca (1974) from sandy soil, Kharga Oasis, Western Desert.

Aspergillus floriformis Samson \& Mouch., Antonie van Leeuwenhoek 41(3): 343 (1975)

Isolated by Samson and Mouchacca (1975) from desert 
soil.

Aspergillus fruticulosus Raper and Fennell, The Genus Aspergillus: 507 (1965)

Isolated as Emericella fruticulosa (Raper \& Fennell) Malloch \& Cain by Samson and Mouchacca (1974) from desert soil, Kharga Oasis.

Aspergillus latus (Thom \& Raper) A.J. Chen, Frisvad \& Samson, in Chen, Frisvad, Sun, Varga, Kocsubé, Dijksterhuis, Kim, Hong, Houbraken \& Samson, Stud. Mycol. 84: 69 (2016)

Isolated as A. nidulans var. latus Thom \& Raper 1939 by Abdel-Fattah et al. (1977) from salt marsh soil and as Emericella nidulans var. lata (Thom \& Raper) Subram. by Moubasher and Abdel-Hafez (1978) from cultivated soil.

Aspergillus multicolor Sappa, Allionia 2: 87 (1954)

Isolated from soils along Idfu-Marsa Alam road at Eastern desert by Abdel-Hafez et al. (1991)

Aspergillus nidulans (Eidam) G. Winter, Rabenh. Krypt. Fl., Edn 2 (Leipzig) 1.2: 62 (1884)

Isolated by Sabet (1935) from different soil types; as $A$. nidulans var. acristatus Abdel-Hafez et al. (1977) from salt marshes and as Emericella heterothallica (Kwon-Chung, Fennell \& Raper) Malloch \& Cain by (Moubasher et al. 2018a) from citrus and grapevine plantations; $E$. nidulans (Eidam) Vuillemin by Ismail et al. (1995) from various substrates; as E. nidulans var. acristata Subram by Moubasher and Abdel-Hafez (1978) from cultivated soil; as E. nidulans var. dentata Subram by (Moubasher et al. 1985) from desert soil; as E. nidulans var. echinulata Godeas by Ibrahim (1999) from cultivated soil; as $A$. heterothallicus Kwon-Chung, Raper and Fennell (1965) by Abdel-Sater et al. (2016) from soil grapevine plantation.

Aspergillus parvathecius Raper and Fennell, The Genus Aspergillus: 509 (1965)

Isolated as Emericella parvathecia (Raper \& Fennell) Malloch and Cain (1972) by Ismail et al. (1995) from various substrates.

Aspergillus purpureus Samson \& Mouch., Antonie van Leeuwenhoek 41(3): 350 (1975)

Isolated as E. purpurea Samson and Mouchacca (1975) by Mouchacca from sandy soil, Kharga Oasis, Western Desert.

Aspergillus quadrilineatus Thom \& Raper, Mycologia 31(6): 660 (1939)

Isolated by Naguib and Mouchacca (1970) from desert soil; as Emericella quadrilineata (Thom \& Raper) (1955) 78 by (Samson and Mouchacca 1974) Moubasher and Moustafa (1970) from cultivated soil.

Aspergillus rugulosus Thom \& Raper, Mycologia 31(6): 660 (1939)

Isolated by Moubasher and Abdel-Hafez (1978) from soil different localities; as Emericella rugulosa (Thom \& Raper) by Moubasher and Moustafa (1970) from cultivated soil.

Aspergillus rugulovalvus Samson and Gams, in Samson \& Pitt (eds), Advances in Penicillium and Aspergillus

Systematics (New York): 49 (1986) [1985]

Isolated from cultivated soil (after El-Abyad 1997).

Aspergillus spelunceus Raper and Fennell, [as 'speluneus'], The Genus Aspergillus: 457 (1965)

Isolated by Abdel-Sater et al. (2016) from citrus and grapevine plantations in the Assiut region.

Aspergillus spinulosporus Hubka, S.W. Peterson \& M. Kolařík, in Hubka, Nováková, Peterson, Frisvad, Sklenář, Matsuzawa, Kubátová \& Kolařík, P1. Syst. Evol. 302(9): 1290 (2016)

Isolated as A. nidulans var. echinulatus Fennell and Raper (1955) by Abdel-Fattah et al. (1977) from salt marsh soil.

Aspergillus stella-maris Zalar, Frisvad \& Samson, Mycologia 100(5): 789 (2008)

Isolated as Emericella stella-maris (Zalar, Frisvad \& Samson) by Moubasher et al. $(2010,2013)$ from the air of orange plantations.

Aspergillus stellatus Curzi, C. r. Accad. Lincei 19: 428 (1934)

Isolated by Moubasher and Abdel-Hafez (1978) from desert soil, Nile valley and coastal area of Mediterranean and as Emericella variecolor Berk. \& Broome by Moubasher and Abdel-Hafez (1978) from cultivated soil.

Aspergillus stellifer Samson \& W. Gams, in Samson \& Pitt (eds), Advances in Penicillium and Aspergillus Systematics (New York): 52 (1986) [1985]

Isolated by Abdel-Sater et al. (2016) from citrus and grape phyllosphere and carposphere in Assuit.

Aspergillus striatus J.N. Rai, J.P. Tewari \& Mukerji, Can. J. Bot. 42(11): 1521 (1964)

Isolated as Emericella striata (Rai, Tewari \& Mukerji) Malloch \& Cain by Ismail et al. (1995) from various substrates. 
Aspergillus sublatus Y. Horie, Trans. Mycol. Soc. Japan 20(4): 481 (1979)

Isolated as Emericella sublata Y. Horie by Ismail et al. (1995) from various substrates.

Aspergillus tetrazonus Samson \& W. Gams, in Samson \& Pitt (eds), Advances in Penicillium and Aspergillus Systematics (New York): 48 (1986) [1985]

Isolated from cultivated soil (after El-Abyad 1997).

Aspergillus unguis (Émile-Weill \& L. Gaudin) Thom \& Raper, Medical Mycology (Philadelphia): 637 (1934)

Isolated by Naguib (1966) from desert soil (IMI 121637); as Emericella unguis Malloch \& Cain by Ismail et al. (1995) from various substrates.

Aspergillus violaceus Fennell \& Raper, Mycologia 47(1): 75 (1955)

Isolated as Emericella violacea (Fennell \& Raper) Malloch \& Cain by El-Kady and Abdel-Hafez (1981) from barley grains.

Aspergillus violaceobrunneus Samson \& W. Gams, in Samson \& Pitt (eds), Advances in Penicillium and Aspergillus Systematics (New York): 53 (1986) [1985]

Isolated from desert soil (after El-Abyad 1997).

\section{Section 19: Ochraceorosei}

Aspergillus funiculosus G. Sm., Trans. Br. mycol. Soc. 39(1): 111 (1956)

Isolated by El-Abyad et al. (1982) from soil.

\section{Section 20: Silvati}

Aspergillus silvaticus Fennell \& Raper, Mycologia 47(1): 83 (1955)

Isolated by El-Hissy et al. (1990) from Aswan high Dam Lake.

\section{Section 21: Sparsi}

Aspergillus conjunctus Kwon-Chung \& Fennell, (in Raper \& Fennell, The Genus Aspergillus: 552 (1965)

Isolated by El-Morsy (1990) from mangrove soil collected from the Red sea.

Aspergillus panamensis Raper \& Thom, Mycologia 36(6): 568 (1944)

Isolated by Abdel-Sater et al. (2016) from soil of citrus and grapevine plantations.

Aspergillus sparsus Raper \& Thom, Mycologia 36(6): 572 (1944)
Isolated by Abdel-Kareem (2010) from deteriorating historical textiles in the Egyptian Museum and the Coptic Museum in Cairo.

\section{Section 22: Raperi}

Aspergillus raperi Stolk \& J.A. Mey., Trans. Br. mycol. Soc. 40(2): 190 (1957)

Isolated by Abdel-Kareem (2010) from deteriorating historical textiles in the Egyptian Museum and the Coptic

Museum in Cairo.

\section{Section 23: Usti}

Aspergillus calidoustus Varga, Houbraken \& Samson, Eukaryotic Cell 7(4): 636 (2008)

Isolated by Moubasher et al. (2016) from soil of citrus plantations.

Aspergillus carlsbadensis Frisvad, Varga \& Samson, in Samson, Varga, Meijer \& Frisvad, Stud. Mycol. 69: 88 (2011)

Isolated by (Moubasher et al. 2018b) from soil of orange and grapevine plantations

Aspergillus deflectus Fennell \& Raper, Mycologia 47(1): 82 (1955)

Isolated by El-Hissy et al. (1990) from Aswan high Dam Lake.

Aspergillus granulosus Raper \& Thom, Mycologia 36(6): 565 (1944) (1944)

Isolated from saline sandy soil (after El-Abyad 1997).

Aspergillus insuetus (Bainier) Thom \& Church, Manual of the Aspergilli: 153 (1929)

Isolated by Sabet (1935) from sandy soil at Burg Al Arab.

Aspergillus minutus E.V. Abbott, Iowa St. Coll. J. Sci. 1(3) (1927)

Isolated by Sabet (1935) from soil of sandy garden, Giza.

Aspergillus porphyreostipitatus Visagie, Hirooka \& Samson, n Samson, Visagie, Houbraken, Hong, Hubka, Klaassen, Perrone, Seifert, Susca, Tanney, Varga, Kocsubé, Szigeti, Yaguchi \& Frisvad, Stud. Mycol. 78: 112 (2014)

Isolated by (Moubasher et al. 2018b) from phyllosphere sample of orange plantations

Aspergillus pseudodeflectus Samson \& Mouch., Antonie van Leeuwenhoek 41(3): 345 (1975)

Isolated by Samson and Mouchacca (1975) from desert soil. 
Aspergillus puniceus Kwon-Chung \& Fennell, in Raper \&Aspergillus spelunceus Raper and Fennell, [as Fennell, The Genus Aspergillus: 547 (1965) 'speluneus'], The Genus Aspergillus: 457 (1965)

Isolated by Naguib and Mouchacca (1970) from desert soil. Isolated from sandy soils (after El-Abyad 1997). Aspergillus versicolor (Vuill.) Tirab., Ann. Bot., Roma 7: Aspergillus ustus (Bainier) Thom \& Church, The 9 (1908)

Aspergilli: 152 (1926)

Isolated by Sabet (1935) from soil.

Aspergillus ustus var. pseudodeflectus (Samson \& Mouch.) Kozak., Mycol. Pap. 161: 131 (1989)

Isolated by Samson and Mouchacca (1975) from desert soil.

\section{Section 24: Versicolores}

Aspergillus humicola Chaudhuri and Sachar, Annls mycol. 32(1/2): 97 (1934)

Isolated by El-Abyad and Migahed (1989) from soil.

Aspergillus peyronelii Sappa, Allionia 2: 248 (1955)

Isolated from sandy soils (after El-Abyad 1997).
Isolated by Moubasher (1966) from soil samples (IMI 114287).

Aspergillus sydowii (Bainier \& Sartory) Thom and Church, The Aspergilli: 147 (1926)

Isolated by Sabet (1939) from loamy field of agriculture college, Cairo University.

\section{Subgenus: Ornati (Problematic taxon)}

\section{Section: Ornati}

Sclerocleista ornata (Raper, Fennell \& Tresner) Subram., Curr. Sci. 41(21): 757 (1972)

Isolated by Abo El-Lel (1977) from salt marshes and as Aspergillus ornatus by Hamed (2016) from farm soil at Al Sharqia Governorate. 


\section{Key to the Aspergillus taxa recorded in Egypt}

\section{Subgeneric classification of Aspergillus taxa in Egypt}

Subgenus Aspergillus - Uniseriate, xerophilic species, conidiophore stipes smooth-walled, hyaline or brownish or greenish. Vesicles slightly inflated to subglobose, fertile in the upper half. Conidial masses mostly in shades of green, yellow cleistothecia.

Subgenus Circumdati - Uniseriate or biseriate, conidiophore stipes pigmented or hyaline, smooth-walled or roughened. Vesicles perfectly globose, fertile over the entire surface. Conidial heads typically radiate, conidial masses variously pigmented.

Subgenus Cremei - Uniseriate or biseriate, conidia en masse grey-green to yellow brown, globose to subglobose, metulae and phialides produced synchronously, except in A. inflatus, where they are produced successively, cream to buff cleistothecia, species are moderately osmophilic and halophilic.

Subgenus Fumigati - Uniseriate, conidiophore stipes smooth-walled, hyaline, greenish or yellow-brown. Vesicles flaskshaped or clavate. Conidial masses either in pale grey-green to dark blue-green or in pinkish fawn shades, white to cream cleistothecia.

Subgenus Nidulantes - Biseriate, conidiophore stipes smooth-walled, hyaline or pigmented. Vesicles subglobose to somewhat clavate, fertile in the upper half. Conidial heads columnar or radiate, variously pigmented. Hülle cells often present, dull yellow to buff cleistothecia, ascospores red to purple.

\section{Subgenus 1: Aspergillus}

\section{Section 1: Aspergillus}

Xerophilic species. Conidiophore stipes smooth-walled, hyaline or brownish. Vesicles dome-like. Metulae absent. Conidial heads radiate to somewhat columnar, typically in shades of green but light brown in one species.

\section{Cleistothecia present}

A. Ascospores $6 \mu \mathrm{m}$ or less along the main axis, conidia less than $7 \mu \mathrm{m}$ in diameter

1. Ascospore equatorial ridges lacking or showing only as traces

a. Ascospore furrow shallow

(1) Conidial surface spinulose A. ruber

(2) Conidial surface verrucose A. tonophilus

(3) Conidial surface microtuberculate $(3.5-5.5 \times 3-4.5) \mu \mathrm{m}$. A. xerophilous

b. Ascospore furrow showing as a slit, conidial surface spinulose

(1) Conidial heads small. A. pseudoglaucus

(2) Conidial heads large. A. repens

2. Ascospore equatorial ridges interrupted

a. Conidiophores, phialides and sub-vesicular area are proliferating

b. Conidiophores, phialides and sub-vesicular area are none proliferating

(1) Smooth, minute rough ornamentation of ascospore convex surface A. proliferans

(2) Smooth to slightly verruculose ornamentation of ascospore convex surface A. glaucus

3. Ascospore equatorial crests well developed

a. Conidial surface smooth. A. chevalieri

b. Conidial surface verrucose A. intermedius

(1) Ascospore crests thick, ascospores $5 \mu \mathrm{m}$ or smaller along the main axis A. montevidensis

(2) Ascospore crests thin and wavy, ascospore size up to $6 \mu \mathrm{m}$. A. cristatus

(3) Ascospore crests short and rigid, valve surface definitely rough to verrucose. 
B. Ascospores $6 \mu \mathrm{m}$ or more along the main axis, conidium diameter $7 \mu \mathrm{m}$ or more

1. Ascospore equatorial ridges lacking or very limited, furrow present.

a. conidial surface verrucose

A. halophilicus

b. conidial surface spiny.

A. umbrosus

2. Ascospore equatorial ridges and furrow present, conidial surface verrucose to spinulose

a. Conidial diameter up to $10 \mu \mathrm{m}$ or more, conidial surface verrucose

(1). Ascospore main axis not greater than $7 \mu \mathrm{m}$.

A. mangini

(2). Ascospore main axis up to $10 \mu \mathrm{m}$.

A. echinulatus

b. Conidial diameter less than $10 \mu \mathrm{m}$

(1) Conidium surface with small scattered spiny processes, ascospore main axis up to $8 \mu \mathrm{m} . .$. A. niveo-glaucus

(2) Thick spines covering the whole conidium surface, ascospore main axis never exceed 7 $\mu \mathrm{m}$

A. leucocarpus

II. Cleistothecia lacking, ascospores borne in naked clustered asci

A. athecius

\section{Section 2: Restricti}

Xerophilic species. Conidiophore stipes smooth-walled, hyaline, sometimes greenish in apical part. Vesicles slightly inflated to subglobose. Metulae absent. Conidial heads definitely or loosely columnar. Conidia cylindrical when young, later ellipsoidal to globose. Conidial masses in pale green shades.

I. Heads columnar, vesicle small, flask shaped, dome-like or only gradual enlargments of the conidiophore apices, fertile on the upper surface only, less than $1.5 \mathrm{~cm}$ at 3 weeks on Czapek's agar.

1. Rapidly growing on M40Y agar, dark olive green; columns long, often twisted....

A. restrictus

2. Less rapidly growing on M40Y agar, light gray green; columns more delicate.

A. conicus

II. Heads radiate when young, tardily becoming loosely or irregularly columnar, vesicle subglobose to pear-shaped, fertile over the upper half to two-thirds. A. penicillioides

\section{Subgenus 2: Circumdati}

\section{Section 3: Assiuti}

Section Assiuti containing species of fast growing, whitish-creamish colonies; not able to grow at $45^{\circ} \mathrm{C}$; with uniseriate, globose to radiate conidial heads; smooth stipes, hyaline and thick-walled vesicles and conidiophore stipes; and pyriform to elongate when young to globose or subglobose conidia at maturity. The section contains only one species, $\boldsymbol{A}$. assiutensis. Fast-growing whitish colonies, uniseriate conidiogenous cells, globose to radiate heads, thick-walled stipes and vesicles, pyriform to elongate conidia when young and globose to subglobose at maturity. For more details please consult Moubasher and Soliman (2011).

\section{Section 4: Candidi}

Section Candidi containing slow growing colonies with globose conidial heads having white to yellowish conidia, conidiophores smooth, small conidiophores common, metulae present and covering the entire vesicle, some large Aspergillus heads with large metulae, presence of diminutive heads in all species, conidia smooth or nearly so with a subglobose to ovoid shape, and the presence of sclerotia.

I. Colony colour white, colony reverse uncoloured to yellow, conidial head globose, smooth conidiophore $(500-1000 \mu \mathrm{m})$, subglobose smooth conidia, no growth at $37^{\circ} \mathrm{C}$, with purple to black sclerotia.....

II. Colony colour sulphur yellow, colony reverse uncoloured, conidial head radiate, smooth conidiophore $(400-800 \mu \mathrm{m})$, ellipsoidal smooth conidia, no growth at $37^{\circ} \mathrm{C}$, no sclerotia A. campestris 


\section{Section 5: Circumdati}

Aspergillus section Circumdati or the Aspergillus ochraceus group, includes species with biseriate conidial heads, rough walled stipes, biseriate conidial heads, yellow to ochre conidia and sclerotia that do not turn black.

I. Conidial heads in pale pure yellow shades

A. Sclerotia cream to pale yellow, produced in a dense layer, $300-450 \mu \mathrm{m}$ in diameter, conidial heads loosely radiate, spore chains adherent into narrow divergent columns............................................ sulphureus

B. Sclerotia white to light orange to yellow, colonies are poorly sporulating after $7 \mathrm{~d}$, conidial heads globose, minor portion elongated and radiate.

A. fresenii

C. Sclerotia white to cream to pale pink, produced singly, 1.0 to $1.5 \mathrm{~mm}$ in diameter, conidial heads hemispherical to loosely columnar or split into two or more compact columns.

A. sclerotiorum

D. Sclerotia light yellow, sparse, $115-550 \mu \mathrm{m}$ in diameter, conidial heads globose, radiate A. bridgeri

E. Sclerotia black white when young, produced centrally, $155-820 \mu \mathrm{m}$ in diameter, conidial heads radiate, globose.

A. robustus

F. Sclerotia only detected on MEA, white, 740-990 × 660-800 $\mu \mathrm{m}$, conidial heads radiate splitting into two columns.

A. roseoglobulosus

G. Sclerotia absent, conidial heads radiating, globose

A. gaarensis

II. Conidial heads in bright golden yellow shades

A. Sclerotia orange to rufous, globose to subglobose, 500-700 $\mu \mathrm{m}$ in diameter, conidia heavy walled, smooth, elliptical or ovate, 3.3-4.4 $\mu \mathrm{m}$ by $2.5-3.0 \mu \mathrm{m}$, conidial heads remaining bright in age.

A. auricomus

III. Conidial heads in dull yellowish cream, buff or ochraceous shades

A. Sclerotia produced in most strains

1. Sclerotia abundant, small, commonly 400 to $500 \mu \mathrm{m}$

a. Sclerotia pure yellow then brown, conidia globose, subglobose or elliptical, 2.75-3.5 $\mu \mathrm{m}$ or $3.0-3.3 \mu \mathrm{m}$ by 2.5 to $2.8 \mu \mathrm{m}$

A. melleus

2. Sclerotia scattered, developing late, large, commonly 500 to $1000 \mu \mathrm{m}$

a. Sclerotia pink to vinaceous purple when mature, globose, ovate to cylindrical, conidia globose to subglobose, mostly 2.5 to $3.0 \mu \mathrm{m}$

A. ochraceus

b. Sclerotia cream to buff or clay colour, globose to ovate, conidia elliptical to pyriform 4.0 to 5.0 um by 3.0 to $3.5 \mathrm{um}$.....

.A. ostianus

c. Sclerotia white to cream, ovate to discoid, conidia ovate to elliptical, mostly 3.2 to 4.0 um by 2.8 to $3.2 \mu \mathrm{m}$

\section{B. Sclerotia Unknown}

A. elegans

1. Colonies close textured, sporulating slowly, conidial heads pinkish buff, conidia subglobose, ovate or elliptical, mostly 3.0 to $4.0 \mu \mathrm{m}$ by 2.5 to $3.0 \mu \mathrm{m}$....

A. petrakii

IV. Conidial heads in light yellow to olive brownish to brown

A. Sclerotia reddish brown, $350-650 \mu \mathrm{m}$

A. flocculosus

B. Sclerotia absent.

A. insulicola

\section{Section 6: Flavi}

Conidiophore stipes hyaline, usually warted. Vesicles clavate, flask-shaped, globose or subglobose, fertile over most of their surface. Metulae present or absent. Conidial heads radiate, conidial masses yellow-green to deep olive-brown.

I. Conidial heads in pale to intense yellow or yellow-green shades when young

A. Heads uniseriate, radiate, conidia prominently echinulate, dark yellow green in mass, sclerotia absent. ....

A. parasiticus

B. Heads biseriate in many conidiophores, radiate or very loosely columnar, conidia finely echinulate, brownish, yellow green in mass, irregularly shaped sclerotia sometimes present.

A. flavus 
II. Conidial heads in bright golden yellow shades to cinnamon

A. Heads biseriate on old conidiophores, uniseriate on small vesicles, columnar to radiate, conidia smooth, oval to subglobose, abundant grey black sclerotia present.

A. alliaceus

III. Conidial heads in deep yellow-green to olive-brown shades when young; conidia conspicuously verruculose.

A. Conidial heads at first deep yellow-green, shifting to brownish green or brown on Czapek's agar....

A. tamarii

B. Conidial heads quickly olive-brown then dark brown

A. flavofurcatus

IV. Conidial heads in pale yellowish olive or grayish olive shades; conidia smooth or nearly so.

A. Conidiophores conspicuously echinulate

A. subolivaceous

B. Conidiophores smooth or nearly so

A. avenaceus

\section{Section 7: Flavipedes}

Aspergillus section Flavipedes contains species found worldwide in soils and rhizospheres, indoor and cave environments, as endophytes, food contaminants and occasionally as human pathogens.

I. Colonies on MEA and CYA at $25 \mathrm{C}$ after $14 \mathrm{~d}$ brightly yellow

A. Ascospores develop after 3-4 wk of cultivation on MEA at $25^{\circ} \mathrm{C}$.

A. neoflavipes

II. Colonies on MEA and CYA at $25{ }^{\circ} \mathrm{C}$ after $14 \mathrm{~d}$ otherwise colored

A. No or very restricted (\#2 mm) growth on CYA at $40{ }^{\circ} \mathrm{C}$ after $7 \mathrm{~d}$, vesicles predominantly spathulate, no production of Hülle cells on MEA.

A. flavipes

\section{Section 8: Jani}

Section Jani species produce three types of conidiophores and conidia, and colonies have green and white sectors making them distinctive.

I. Conidial heads of two colors; green or white, vesicles of green and white heads dissimilar, vesicles of white heads conspicuously clavate, 45 to $60 \mu \mathrm{m}$ by 15 to $18 \mu \mathrm{m}$, borne on long conidiophores usually exceeding $2 \mathrm{~mm}$ in length, smaller dark green heads borne upon short conidiophores with typically ovate vesicles....

A. janus

\section{Section 9: Nigri}

The black aspergilli (Aspergillus section Nigri; Gams et al. 1986) are an important group of species in food mycology, medical mycology and biotechnology. Many species cause food spoilage, but on the other hand are also used in the fermentation industry to produce hydrolytic enzymes, such as amylases or lipases, and organic acids, such as citric acid and gluconic acid (Varga et al. 2000). Conidiophore stipes smooth and hyaline or pigmented below the vesicle. Vesicles globose or nearly so, sometimes dark brown. Metulae present or absent, often pigmented. Conidial heads typically radiate (in some species in divergent columns), conidial masses in shades of black.

I. Sterigmata in two series (biseriate)

A. Colonies (conidial heads) on Czapek's agar appearing hair brown to dark blond, dark brown to carbon black

1.Conidia 6 to $10 \mu \mathrm{m}$ or more in diameter

a. Conidia 7 to $11 \mathrm{um}$ in diameter, globose, very rough

A. carbonarius

b. Conidia 6 to $8 \mathrm{um}$ in diameter, globose, conspicuously roughened with prominent color bars....

c. Conidia 6 to $8.5 \mu \mathrm{m}$ in diameter, globose to subglobose, conspicuously

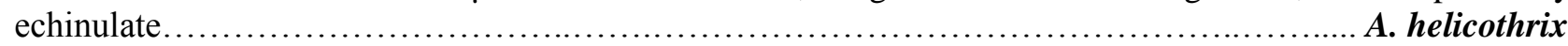

d. Conidia (5.1-) 6-8 (-9.5) 6 (4.8-) 5.8-7.8 (-8.5) $\mu \mathrm{m}$ in diameter, subglobose, conspicuously echinulate when young, becoming verruculose when mature.

A. sclerotiicarbonarius 
2. Conidia $5 \mu \mathrm{m}$ or less in diameter at maturity

a. Conidiophores not exceeding $4 \mathrm{~mm}$ in length

(1) Conidia 3.5-4 $\mu \mathrm{m}$ in diameter, globose, irregularly roughened when young, becoming horizontally flattened and longitudinally striate at maturity. A. ficuum

(2) Conidia 4-5 $\mu \mathrm{m}$ in diameter, globose, irregularly roughened with conspicuous ridges and echinulations not arranged as longitudinal striation.

b. Conidiophores commonly exceeding $5 \mathrm{~mm}$ in length

(1) Conidiophore reaching $1 \mathrm{~cm}$ but also with shorter stalk bearing diminutive heads, conidia globose to subglobose, 3.5-0.5 $\mu \mathrm{m}$, smooth to definitely verruculose.

A. pulverulentus

(2) Conidiophore 0.3 to $1.2 \mathrm{~cm}$, conidia subglobose, 3.5-4.1 x 3.4-3.9 $\mu \mathrm{m}$, usually smooth to finely

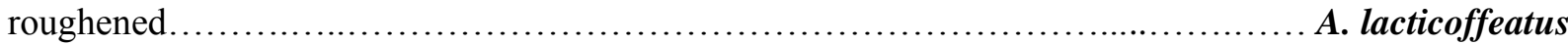

(3) Conidiophore 0.7 to $1.7 \mathrm{~cm}$, conidia subglobose, 3.5-4.8 $\mu \mathrm{m}$ in diameter, echinulate...

(4) Conidiophore 1.0 to $1.7 \mathrm{~cm}$, conidia globose to subglobose, $(3.1-) 3.5-4.3(-4.5) \mu \mathrm{m}$ in diameter, smooth when young, becoming distinct rough walled.... A. costaricensis

B. Colonies (conidial heads) grayish olive brown or deep olive brown when young; usually becoming reddish brown to brownish black, but with olive or grayish colours often persistent.

1. Heads quickly dark black-brown or reddish-brown

a. Heads quickly dark black-brown; colony reverse uncoloured; conidiophores mostly 2 to $3 \mathrm{~mm}$ but up to $5 \mathrm{~mm}$ long; conidia mostly 3.0 to $3.5 \mu \mathrm{m}$ in diameter.

A. tubingensis

b. Heads quickly reddish brown; colony reverse in similar shades; conidiophores usually 1 to $1.5 \mathrm{~mm}$ long; conidia mostly 4.0 to $4.5 \mu \mathrm{m}$ in diameter.

A. awamori

2. Heads persistently dark greyish brown or olive brown

a. Conidia at maturity elliptical, conspicuously echinulate, 5.0 to 5.5 by 3.3 to $3.8 \mu \mathrm{m}$

A. ellipticus

b. Conidia at maturity globose or nearly so, sometimes elliptical when young

(1) Conidia at maturity conspicuously spinulose

A. heteromorphus

(2) Conidia at maturity irregularly and finely roughened. A. foetidus

(3) Conidia at maturity rough to finely echinulate.... A. vadensis

II. Sterigmata uniseriate

A. Conidia globose to subglobose, conspicuously echinulate; vesicle commonly 20 to $35 \mu \mathrm{m}$ but ranging from 15 to $45 \mu \mathrm{m}$

B. Conidia subglobose to definitely elliptical, conspicuously echinulate

1. 3.5-4.0 $\mu \mathrm{m} \times 4.5-05 \mu \mathrm{m}$, vesicle commonly 60 to $80 \mu \mathrm{m}$ but ranging from 35 to $100 \mu \mathrm{m}$

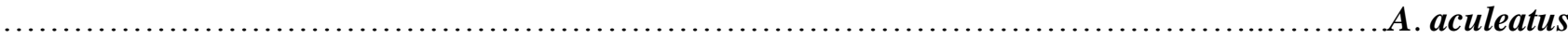

2. $2-4$ x 2.3-4.4 $\mu \mathrm{m}$, vesicle commonly 55 to $65 \mu \mathrm{m}$ but ranging from 43 to $82 \mu \mathrm{m}$ A. aculeatinus

C. Conidia typical ellipsoidal to fusiform conidia, coarsely roughened to echinulate; vesicle 10 to $18 \mu \mathrm{m}$ wide.

.A. violaceofuscus

\section{Section 10: Terrei}

Aspergillus section Terrei includes species with columnar conidial heads in shades of buff to brown. The most important species of this section is A. terreus, which is an ubiquitous fungus in our environment. Strains of this cosmopolitan species are frequently isolated from desert and grassland soils and compost heaps, and as contaminants of plant products like stored corn, barley and peanuts. Conidiophore stipes smooth-walled, hyaline. Vesicles hemispherical. Metulae present. Conidial heads compactly columnar, conidial masses buff, cinnamon, to orange-brown. Hülle cells absent, but globose to ovate, relatively heavy-walled hyaline cells formed from submerged hyphae. 
I. Colonies velvety, conidial heads long, compactly columnar, in cinnamon to orange brown or brown shades; born on short conidiophores

A. Sclerotium-like masses of swollen, relatively heavy-walled cells lacking on MEA

A. terreus

II. Colonies floccose, aerial mycelium conspicuously golden yellow, conidial heads small, compactly columnar, cream to buff; born on conidiophores $500 \mu \mathrm{m}$ or more long.

A. aureoterreus

III. Conidiophores unpigmented or very faintly yellowed

A. Conidia heads at first white, becoming vinaceous fawn, conidia globose to subglobose, smooth, 2.4 to $2.8 \mu \mathrm{m}$ rarely exceeding $3.2 \mu \mathrm{m}$, irregular hyphal branching may occur.

A. carneus

B. Conidia heads lemon yellow to lemon chrome, conidia globose to subglobose, smooth, 2.0 to $2.5 \mu \mathrm{m}$, crusts of Hülle cells recorded.

A. neoniveus

\section{Subgenus 3: Cremei}

\section{Section 11: Cremei}

Conidiophore stipes mostly hyaline, smooth-walled. Vesicles large, globose, fertile over the entire surface. Metulae present or absent, sometimes on the same vesicle. Conidial heads loosely radiate, conidial masses buff-brown, pale yellow-green or blue-green.

I. Ascocarp present

A. Conidia typically barrel to elliptical or occasionally subglobose, cleistothecia cream to yellowish, ascospores 6.0 to $7.0 \mathrm{um}$ by 4.0 to $4.5 \mu \mathrm{m}$, with sharp spines on convex surface, with two wide equatorial

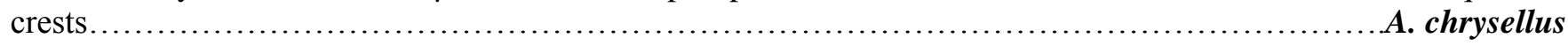

B. Conidia ovate to pyriform but varying from cylindrical to subglobose, cleistothecia cream to buff, ascospores 6.6 to 7.7 by 4.5 to $5.0 \mu \mathrm{m}$, with two prominent equatorial crests, convex surfaces ornamented with few hyaline spikelike extensions.

A. cremeus

II. Ascocarp absent

A. Heads biseriate

1. Conidia up to $6 \mu \mathrm{m}$, globose to broadly ellipsoid, smooth.

A. wentii

2. Conidia up to $4 \mu \mathrm{m}$, globose, echinulate.

3. Conidia up to $4 \mu \mathrm{m}$, subglobose to globose, rarely ovate, very delicately roughened.

4. Conidia up to $4.5 \mu \mathrm{m}$, globose, slightly roughened

A. dimorphicus

A. sepultus

B. Heads mostly uniseriate occasionally biseriate

1. Conidia mostly subglobose, varying from globose to slightly ellipsoidal, hyaline, some what roughened, mostly 4.5 to $6.0 \mu \mathrm{m}$ by 4 to $5 \mu \mathrm{m}$, occasionally up to $7 \mu \mathrm{m}$ in long axis.

A. flaschentraegeri

\section{Subgenus 4: Fumigati}

\section{Section 12: Cervini}

Conidiophore stipes smooth-walled, yellow-brown. Vesicles globose to flask-shaped. Metulae absent. Conidial heads radiate

I. Conidiophores usually 100 to $300 \mu \mathrm{m}$ in length, walls very thick, $1.5-2.0 \mu \mathrm{m}$, heads erect.................A. cervinus

II. Conidiophores not exceeding $100 \mu \mathrm{m}$ in length, vescicle upright or borne at angel......................A. parvulus

\section{Section 13: Clavati}

Conidiophore stipes tall, thick-walled, smooth-walled, hyaline. Vesicles long clavate. Metulae absent. Phialides covering the entire vesicle. Conidial heads large, splitting into compact columns, conidial masses in blue-green shades.

I. Conidial structures often 1 to 5 or more $\mathrm{cm}$ in length

A. giganteus

II. Conidial structures not exceeding $4.0 \mathrm{~mm}$ in length.

III. Conidial structures less than $1.0 \mathrm{~mm}$ in length. 


\section{Section 14: Fumigati}

Conidiophore stipes smooth-walled, often greenish. Vesicles flask-shaped to clavate. Metulae absent. Phialides confined to the apical part, parallel. Conidial masses compactly columnar, commonly grey-green to dark blue-green.

I. Ascocarp absent

A. Conidial heads erect, compact, strongly to loosely columnar, vesicle commonly 15 to $30 \mu \mathrm{m}$ in diameter, upright on the conidiophore

1. Conidiophores $0.5 \mathrm{~mm}$ or less, conidial head dark green, conidia globose, echinulate

A. fumigatus

2. Conidiophores $0.08 \mathrm{~mm}$, conidial head gray-turquoise to gray-green, conidia subglobose, ovoid and smooth.

A. turcosus

B. Conidial heads often presenting a nodding appearance, smaller than the preceding and not consistently columnar; vesicles less than $20 \mu \mathrm{m}$ in diameter.

1. Conidiophores thin walled, sinuous, vesicles uncoloured, and often strongly nodded, conidia in pale blue-green shades

A. viridinutans

2. Conidiophores heavy walled, vesicles and sterigmata coloured, conidia in dark blue-green shades

a. Conidia conspicuously echinulate, colony reverse uncoloured or nearly so................... A. duricaulis

b. Conidia finely spinulose, colony reverse in reddish brown to deep rose shades.

A. brevipes

II. Ascocarp present

A. Cleistothecia and enveloping hyphae white to cream in colour

1. Heterothallic

a. Convex surfaces of ascospores distinctly cerebriform.

A. fennelliae

2. Homothallic

a. Convex surfaces bearing anastomosing ridges to give a large and somewhat irregular reticulation....

A. fischeri

\section{Subgenus 5: Nidulantes}

\section{Section 15: Aenei}

Brown conidiophores, ampulliform vesicle, biseriate, smooth convex face ascospores with two crests, no growth at $40^{\circ} \mathrm{C}$.

\section{Aspergillus bicolor}

Conidial heads, dark green, columnar, up to 200-300 X 80-120 $\mu \mathrm{m}$, biseriate; conidiophores brown pigmented, smooth; vesicles globose or nearly so, brown, 10.5-24.0 $\mu \mathrm{m}$ in diam, fertile over the upper two-thirds; conidia subglobose to ellipsoidal, mostly 3.5-4.0 x 2.5-3.2 $\mu \mathrm{m}$ rarely to $4.5 \times 3.5 \mu \mathrm{m}$, bright green, roughened. Hülle cell present. Cleistothecia globose, with a reddish, ascospores maturing slowly, orange red, lenticular with very low equatorial crests, convex walls smooth to delicately echinulate.

\section{Section 16: Bispori}

\section{Aspergillus bisporus}

Species is characterized by production of two conidial heads in low water activity medium (DG18), conidial heads with uniseriate phialides bearing short chains of large, globose, black, coarsely dentate conidia. The second type of conidial structure long, light-olivish conidial chains consisting of smooth to slightly rough, globose to elliptical conidia.

\section{Section 17: Cavernicolus}

I. Long conidiophores, 9-35 x 1.9-2.5 $\mu \mathrm{m}$, vesicle globose 3.0-8.5 $\mu \mathrm{m}$ in diameter, conidia globose to subglobose, smooth, 2.7-04 $\mu \mathrm{m}$ in diameter, hülle cells globose to subglobose. A. egyptiacus

II. Short conidiophores, 5-12 x 2.2-2.5 $\mu \mathrm{m}$, vesicle irregular in shape 2.5 (3.5-4.5) $6.0 \mu \mathrm{m}$ in diameter, conidia globose to subglobose, irregularly roughened, $2.5-4.0 \mu \mathrm{m}$ in diameter, hülle cells globose to subglobose or elongate with ends more or less pointed.. A. subsessilis 


\section{Section 18: Nidulantes}

Conidiophore stipes brown, smooth-walled, commonly less than $250 \mu \mathrm{m}$ long. Vesicles hemispherical to flask-shaped. Metulae present, covering the upper half of the vesicle. Conidial heads typically columnar, conidial masses in green shades. Hülle cells typically abundantly produced, globose to irregularly ovate or pyriform.

\section{Cleistothecia present}

A. Ascospores lenticular, non-stellate

1. Ascospores, orange-red, reddish brown in color

a. Convex surfaces are smooth, two equatorial crests

(1) Light orange, $4-5 \times 3.5-4.5 \mu \mathrm{m}$, crests $0.8-1 \mu \mathrm{m}$

A. aurantiobrunneus

(2) Orange to reddish brown, $4.5-5.5 \times 3-5 \mu \mathrm{m}$, crests $0.8-1 \mu \mathrm{m}$ A. fruticulosus

(3) Orange to reddish brown, 3.5-5 × 3-4.5 $\mu \mathrm{m}$, crests $0.5-1$ (entire or dentate)... A. nidulans

(4) Light orange, orange or reddish brown, 3.5-5 × 3-5 $\mu \mathrm{m}$, incompletely reticulate or ribbed, crests 1-1.5 $\mu \mathrm{m}$

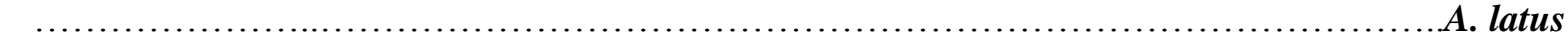

(5) Orange to reddish brown 4-4.5 × 3-4.5 $\mu \mathrm{m}$, crests $0.5-1$ (entire, defective or with irregular protuberance) A. quadrilineatus (三 A. tetrazonus)

(6) Light orange, orange or reddish brown, convex surfaces smooth, incompletely reticulate or ribbed, globose to subglobose, $3.5-5 \times 3-5 \mu \mathrm{m}$; in side view lenticular, with two pleated equatorial crests measuring $1-1.5$ $\mu \mathrm{m}$. .....

A. sublatus

b. Convex surfaces are smooth, four equatorial crests

(1) Reddish brown, 4 crests, two of them are equatorial, conspictous, pleated and about 0.5 um wide, the other two are in a subequatorial position and only seen under SEM, 3.2-3.8 $\times 2.5-2.8 \mu \mathrm{m}$

A. parvathecius

c. Convex surfaces are tuberculate, reddish brown, 6.5-7.5 $\times 6-7.5 \mathrm{um}$, crests $0.5 \mu \mathrm{m}$

A. desertorum

d. Convex surfaces are roughened, bearing simple or anastomosing thickenings arranged in more or less concentric rings, orange, $6-7 \times 5-5.5 \mu \mathrm{m}$; in side view broadly lenticular. A. striatus

e. Convex surfaces are echinulate, $3.5-4.5 \times 3-4.5 \mu \mathrm{m}$; with two pleated equatorial crests measuring $0.8-1$ $\mu \mathrm{m}$.

A. spinulosporus

2. Ascospores orange, greyish violet, reddish purple or brownish red

a. Spore body 4-4.5 $\times \quad 3.5-4 \mu \mathrm{m}$, convex surfaces are rugulose, crests $0.5-0.6$ $\mu \mathrm{m}$.

3. Ascospores brown

a. Spore body $6-7 \times 4.5-5 \mu \mathrm{m}$, crests $0.3-0.6 \mu \mathrm{m}$

4. Ascospores violet

a. Spore body 4-6.5 × 3-5 $\mu \mathrm{m}$, convex surfaces roughened, with reticulate intertwined ornamentation, low equatorial crest, less than $0.3 \mu \mathrm{m}$ wide.

A. violaceus ( $\equiv$ A. violaceobrunneus)

B. Ascospores stellate

1. Ascospore size $13-16 \mu \mathrm{m}$, spore body $3-4.5 \times 2.5-4.5 \mu \mathrm{m}$

A. stella-maris

2. Ascospore size $10-14 \mu \mathrm{m}$, spore body $3.5-4 \times 3-4 \mu \mathrm{m}$ A. stellatus (= A. stellifer)

II. Cleistothecia absent

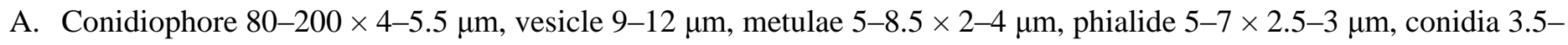
$5 \mu \mathrm{m}$, green in mass

A. aureolatus

B. Conidiophore 200-300 × 3-6 $\mu \mathrm{m}$, vesicle 10-15 um, metulae 5-8 8 3-3.5 $\mu \mathrm{m}$, phialide $6.5-8 \times 3-4.5 \mu \mathrm{m}$, conidia 3-4 $\mu \mathrm{m}$, green in mass

.A. caespitosus

C. Conidiophore $150 \times 5.5-7 \mu \mathrm{m}$, vesicle $11-15 \mu \mathrm{m}$, metulae 9-11 $\times$ 4-5 $\mu \mathrm{m}$, phialide $6-8 \times 4-6 \mu \mathrm{m}$, conidia 3.5-4.7 $\mu \mathrm{m}$, green in mass A. floriformis

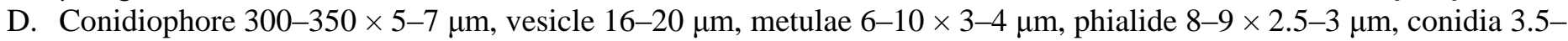
$5.5 \mu \mathrm{m}$ A. multicolor

E. Conidiophore 130-300 × 4-6 $\mu \mathrm{m}$, vesicle 7-11 $\mu \mathrm{m}$, metulae 4-6.5 × 2.5-3.5um, phialide 5.5-7.5 $\times 2-2.5 \mu \mathrm{m}$, conidia $2.5-3.5 \mu \mathrm{m}$, blue green in mass. 
F. Conidiophore 50-100 × 3-5 $\mu \mathrm{m}$, vesicle $8-10 \mu \mathrm{m}$, metulae 5-7 $\times 2.5-3.5 \mu \mathrm{m}$, phialide 5-9 $\times 2-2.5 \mu \mathrm{m}$, conidia $2.5-4 \mu \mathrm{m}$

A. unguis

\section{Section 19: Ochraceorosei}

Section containing species not able to grow at $37{ }^{\circ} \mathrm{C}$, producing yellow ellipsoidal conidia, biseriate conidial heads, long conidiophore stipes that are smooth. Only one recorded problematic species in Egypt.

\section{Section 20: Silvati}

Section containing species, producing conidial structures in deep forest green shades near dusky yellow green; reverse in buff to flesh pink shades; exudate limited, colorless. Conidial heads in rich yellow green shades, long brown conidiophores.

I. Conidia heads of one colour, vesicles globose to somewhat elongate, globose to subglobose Hülle cell often present, mature conidia not exceeding $4.0 \mu \mathrm{m}$, consistently globose to subglobose, conidiophores definitely brown, smooth, radiate conidial head, very dark yellow-green.

A. silvaticus

\section{Section 21: Sparsi}

Aspergillus section Sparsi includes species which have large globose conidial heads with colours ranging from light grey to olive-buff. Vesicles globose, fertile over the entire surface. Metulae usually present. Conidial heads globose, radiate to irregularly split.

I. Conidia are produced sparsely on all media, conidia 3-4(5.5) $\mu \mathrm{m}$, globose to very broadly ellipsoidal, smooth to very finely roughened, no Hülle cell formed

A. sparsus

II. Conidia are produced on all media

A. Reddish brown conidial heads, conidia 2.2-2.6 (2.8) $\mu \mathrm{m}$, globose to subglobose, smooth walled, Hülle cells elongate. A. panamensis

B. Buffy olive conidial heads, conidia $2.5(3.0 \times 3.5) 4.0 \mu \mathrm{m}$, globose to subglobose, smooth to delicately roughened with small unique conical to pyriform connectives, Hülle cells elongate, seldom bent

A. conjunctus

\section{Section 22: Raperi}

Conidial heads light greyish blue-green, loosely columnar to radiate, conidia elliptical sclerotia or compact sclerotium like masses of Hülle cells present.

I. Hülle cell masses present, radiate heads, smooth conidiophores, elliptical conidia 3.0 to 3.8 by 2.0 to $2.5 \mu \mathrm{m}$ A. raperi

\section{Section 23: Usti}

Conidiophore stipes brown, smooth-walled. Vesicles hemispherical. Metulae present. Conidial heads radiate or broadly columnar, conidial masses drab, olivaceous or dull brown.

I. Vesicles upright on the conidiophore

A. Conidial heads in olive-grey to drab or red-brown shades

1. Conidial heads variable, radiate when young to loosely or broadly columnar at maturity

a. Grey to brown colored colony, irregular to elongate hülle cells sometimes present, associated with pigmented mycelium, rough walled globose conidia.

A. ustus

b. Drab colored colony, elongate hülle cells abundantly produced, forming conspicuous masses associated with bright pigmented yellow mycelium, globose conidia, spinulose, to finely roughened

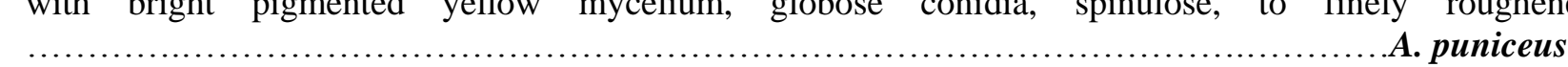

B. Conidial heads pale blue-green in color, hülle cells abundantly produced, irregularly globose, ovoid or somewhat elongated, conidia globose delicately echinulate. 
C. Conidial heads yellow brown with white tufts of conglomerates of Hülle cells. Conidiophores smooth, brown, 4-5 $\mu \mathrm{m}$ wide, vesicles globose, 10-14 $\mu \mathrm{m}$ in diam., conidia distinctly ornamented with spines or warts, ellipsoidal 2.5-3.0 × 3.0-3.5 $\mu \mathrm{m}$.

A. carlsbadensis

D. Conidial heads yellow brown blond/grayish yellow, brownish gray or grayish brown, hyphae inconspicuous, conidiophore short 150 to $300 \mu \mathrm{m}$ (minimum, $130 \mu \mathrm{m}$ ), smooth, brown; vesicles 9 to $15 \mu \mathrm{m}$ (range, 7 to 20 $\mu \mathrm{m})$ wide, pyriform to broadly spathulate; conidia globose 2.7 to $3.5 \mu \mathrm{m}$, very rough ornamentation ( 0.5 to 0.8 $\mu \mathrm{m}$ high), inner and outer wall visible. Hülle cells sparsely produced, irregularly elongated, in scattered groups... A. calidoustus

E. Conidial heads brown

1. Vesicles 11-16 $\mu \mathrm{m}$ in diameter, conidia globose, $5 \mu \mathrm{m}$ in diameter, echinulate, dark brown...... A. insuetus

2. Vesicles 8.0 to $18.0 \mu \mathrm{m}$ in diameter, conidia globose, 3.2 to $4.5 \mu \mathrm{m}$ in diameter, verrucose, light brown

A. minutus

II. Vesicles borne at a sharp angle to the vertical axis of the conidiophore

A. Conidiophore long (40 to $50 \mu \mathrm{m}$ ) in some strains up to $125 \mu \mathrm{m}$ in others, smooth, brown, conidia globose to subglobose, 3.0-3.5 $\mu \mathrm{m}$ in diam, with variable ornamentation, smooth when young to irregularly

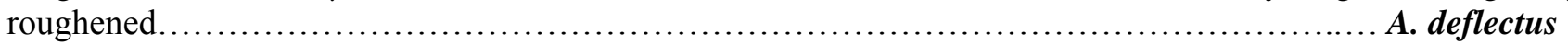

B. Short conidiophore, curved, rough-walled with warty protuberances, brown, conidia globose to ellipsoidal, thick-walled, brown, ornamented with small warts and colour bars, 3.5-4.0 $\mu \mathrm{m}$ in diam. Hulle cells

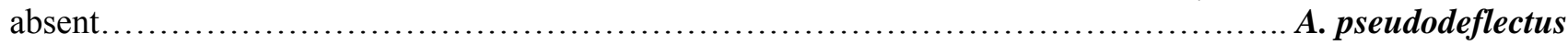

III. Penicillium-like conidiophores present, conidiophores reddish brown, hyaline also present, mostly smooth, some areas contain warts, vesicles globose, sometimes slightly elongated; conidia globose to subglobose, often covered by a thick layer, rough, Hülle cells produced on OA; Sclerotia absent....

A. porphyreostipitatus

\section{Section 24: Versicolores}

Conidiophore stipes smooth-walled, hyaline or pale brown, mostly $>300 \mu \mathrm{m}$ long. Vesicles ovate to ellipsoidal. Metulae present, covering the upper half to three quarters of the vesicle. Conidial heads radiating or loosely columnar, conidial masses usually in some shades of green. Hülle cells usually abundant, globose.

I. Vesicles globose to somewhat elongate, fertile over most of the vesicular surface; globose to subglobose Hülle cells often present, compact hyphal masses and sclerotia lacking

A. Mature conidia not exceeding $0.4 \mu \mathrm{m}$, consistently globose to subglobose

1. Conidiophores uncoloured to faintly yellowish

a. Conidial heads variable in colour, light yellow green, buff to orange-yellow, or occasionally flesh coloured. A. versicolor

b. Conidial heads always blue-green when young. A. sydowii

2. Conidiophores definitely brown, smooth walled; conidial heads variable in shape, often loosely columnar.

A. spelunceus

II. Vesicles turbinate, often borne at a slight angle to the conidiophore, conidial head dark yellow-green; conidia globose, minutely asperulate, $2.5-3.0 \mu \mathrm{m}$ in diameter, true sclerotia present, cream to buff. A. peyronelii

\section{Problematic taxa}

\section{Section: Ornati}

Sclerocleista ornata (Raper, Fennell \& Tresner) Subram (1972)

A. alutaceus Berk. \& M.A. Curtis (1875)

A. funiculosus G. Sm. (1965)

A. humicola Chaudhuri and Sachar (1934)

A. koningii Oudem. (1902)

A. neoellipticus Kozak (1989)

A. olivaceus Delacr (1897)

A. reptans Samson \& W. Gams (1986)

90 
A. sacchari Chaudhuri and Sachar (1934)

A. terreus Thom var. terreus (1918)

A. terricola Marchal \& É.J. Marchal (1921)

A. thomii G. Sm. (1951)

\section{Conclusion}

The present work is considered the first list of genus Aspergillus in Egypt recovered from; soil (cultivated, salt marsh, desert, reclaimed), plants, insects, air, seaweeds, herbivore dung, mangroves, aquatic habitats, stored seeds and grains, deteriorated archeological woods, plant roots and dairy products. Subgenus Polypaecilum not recorded till now in Egypt. Therefore, it should be kept in mind that, although the present study will add some new data to our information concerning the Egyptian fungi, this updated check-list must be considered as a provisional one always waiting for continuous supplementation. Finally, we suggested nomenclature and classification of the Genus Aspergillus in Egypt into two subgenera as follow:

1. Anamorphic subgenus Aspergillus I contains $\mathbf{1 1}$ sections that have unknown teleomorphs up till now namely: Assiuti, Bispori, Cavernicolus, Cervini, Candidi, Jani, Ochraceorosei, Raperi, Silvati, Sparsi and Versicolores.

2. Teleomorphic subgenus Aspergillus II contains 14 sections that have teleomorphs, recently updated, namely: Aenei, Aspergilli, Circumdati, Clavati, Cremei, Flavi, Flavipedes, Fumigati, Nidulantes, Nigri, Ornati, Restricti, Terrei and Usti.

\section{Conflict of interest}

The authors have no conflicts of interest to declare. All co-authors have seen and agree with the contents of the manuscript.

\section{Acknowledgments}

All thanks to the late professor Abdel Al H. Moubasher (Botany and Micerobiology Department, Faculty of Science, Assiut University) for his invaluable work on Aspergillus since 1960, the late professor Samy M El-Abyad (Botany Department, Faculty of Science, Cairo University) for his courage in documenting the Egyptian fungi in 1997. We express our appreciation to the late professor John C Krug (Centre for Biodiversity and Conservation Biology, Royal Ontario Museum, Toronto) for providing some unavailable papers. We are really indebted to Professor Jean Mouchacca (Laboratoire de Cryptogamie, Muséum National d'Histoire Naturelle, Paris) for his discoveries on Aspergillus in Egypt and his unlimited support all the time.

\section{Reference}

Abdel-Azeem A (1991) Effect of overgrazing on vegetation, microbes and soil in Ismailia-desert habitat. In: Biological Diversity Symposium, MadridSpain. pp 241-246.

Abdel-Azeem A (2009) A preliminary study on diversity of fungi in the World Heritage site of Saint Katherine, Egypt. Assiut Univ J Bot 38:29-54.

Abdel-Azeem A, Rashad H (2013) Mycobiota of outdoor air that can cause asthma: a case study from Lake Manzala, Egypt. Mycosphere 4:1092-1104. https://doi.org/10.5943/mycosphere/4/6/7

Abdel-Azeem A, Salem F (2013) A checklist of Egyptian fungi: I. Protozoan fungal analogues. Mycosphere 4:794-807. https://doi.org/10.5943/mycosphere/4/4/15

Abdel-Azeem AM (2003) Ecological and Taxonomical Studies on ascospore-producing fungi in Egypt. Ph.D. Thesis, Faculty of Science, Suez Canal university,
Egypt.

Abdel-Azeem AM (2010) The history, fungal biodiversity, conservation, and future perspectives for mycology in Egypt. IMA Fungus 1:123-142.

Abdel-Azeem AM, Abdel-Moneim TS, Ibrahim ME, et al (2007) Effect of long-term heavy metal contamination on diversity of terricolous fungi and nematodes in Egypt- a case study. Water, Air, Soil Pollut 186:233254.

Abdel-Azeem AM, El-Morsy EM, Nour El-Dein MM, Rashad HM (2015) Occurrence and diversity of mycobiota in heavy metal contaminated sediments of Mediterranean coastal lagoon El-Manzala, Egypt. Mycosphere 6:228-240. https://doi.org/10.5943/mycosphere/6/2/12

Abdel-Azeem AM, Held BW, Richards JE, et al (2019) Assessment of biodegradation in ancient archaeological wood from the Middle Cemetery at 
Abydos, Egypt. PLoS One 14:1-17. https://doi.org/10.1371/journal.pone.0213753

Abdel-Azeem AM, Ibrahim ME (2004) Diversity of terrophilous mycobiota of Sinai. Egypt J Biol 6:2131.

Abdel-Azeem AM, Salem FM, Abdel-Azeem MA, et al (2016) Biodiversity of the Genus Aspergillus in Different Habitats. In: New and Future Developments in Microbial Biotechnology and Bioengineering: Aspergillus System Properties and Applications. Elsevier, Amsterdam, pp 3-28.

Abdel-Fattah HM, Moubasher AH, Abdel-Hafez SI (1977) Studies on mycoflora of salt marshes in Egypt. I. sugar fungi. Mycopathologia 61:19-26. https://doi.org/10.1007/BF00440754

Abdel-Hafez AI, Abdel-Hafez S 1. I, Mohawed SM, Said AHM (1991) Composition, occurrence and cellulolytic activities of fungi inhabiting soils along Idfu-Marsa Alam road at Eastern desert, Egypt. Bull Fac Sci Assiut Univ 20:21-48.

Abdel-Hafez AII, Abdel-Hafez SII, Mohawed SM (1990a) Fungus flora of air, root and leaf surface of lentil plant cultivated in Upper Egypt. Bull Fac Sci Assiut Univ 19:65-88.

Abdel-Hafez AII, Mazen MB, Galal AA (1989a) Keratinophilic and cycloheximide resistant fungi in soils of Sinai Governorate, Egypt. Cryptogam Mycol 10:265-275.

Abdel-Hafez AII, Mazen MB, Galal AA (1989b) Some ecological studies of osmophilic and halophilic soil fungi of Sinai Peninsula, Egypt. Sohag Bull Pure Appl Sci 5:67-83.

Abdel-Hafez AII, Mazen MB, Galal AA (1990b) Glycophilic and cellulose-decomposing fungi from soils of Sinai Peninsula, Egypt. Arab Gulf J Sci Res 8:153-168.

Abdel-Hafez S, El-Said A (1997) Effect of garlic, onion and sodium benzoate on the mycoflora of pepper, cinnamon and rosemary in Egypt. Int Biodeterior Biodegrad 39:39(1): 67-77.

Abdel-Hafez S, El-Said A, Gherbawy Y (1995a) Mycoflora of leaf surface, stem, bagasse and juice of adult sugarcane (Saccharum officinarum L.) plant and cellulolytic ability in Egypt. Bull Fac Sci Assiut Univ 24:113-130.

Abdel-Hafez S, Ismail M, Hussein N, Abdel-Hameed N (2012) Fusaria and other fungi taxa associated with rhizosphere and rhizoplane of lentil and sesame at different growth stages. Acta Mycol 47:35-48.

Abdel-Hafez S, Moharram A, Abdel-Sater M (2000) Monthly variations in the mycobiota of wheat fields in El-Kharga Oasis, Western Desert, Egypt. Bull Fac Sci Assiut Univ 29:195-211.
Abdel-Hafez S, Moubasher A, Shoreit A, Ismail M (1990c) Fungal flora associated with combine harvester wheat and sorghum dusts from Egypt. J Basic Microbiol 30:467-479. https://doi.org/doi:10.1002/jobm.3620300702

Abdel-Hafez SI, Moubasher AH, Abdel-Fattah HM (1977) Studies on mycoflora of salt marshes in Egypt. IV=osmophilic fungi. Mycopathologia 62:143-151. https://doi.org/10.1007/BF00444107

Abdel-Hafez SII (1974) Ecological studies on Egyptian soil fungi. Faculty of Science, Assiut University, Egypt

Abdel-Hafez SII, El-Maghraby OMO (1993) Thermophilic and thermotolerant fungi of Wadi-Bir-El-Ain soils. Eastern desert, Egypt. Yarmouk Res Basic Eng Sci Ser 2:55-66.

Abdel-Hafez SII, El-Said A, Gherbawy YA (1995b) Seasonal variations of air, leaf and stem surface of sugarcane and amylolytic ability in Egypt. Bull Fac Sci Assiut Univ 24:113-130.

Abdel-Hafez SII, Moubasher AH, Barakat A (1990d) Keratinophilic fungi and other moulds associated with air-dust particles from Egypt. Folia Microbiol (Praha) 35:311-325. https://doi.org/10.1007/BF02821283

Abdel-Hafez SII, Shoreit AAM, Abdel-Hafez AII, E.Maghraby OMO (1986) Mycoflora and mycotoxinproducing fungi of air-dust particles from Egypt. Mycopathologia 93:25-32.

Abdel-Kader MIA, Moubasher AH, Abdel-Hafez SII (1979) Survey of the mycoflora of barley grains in Egypt. Mycopathologia 68:143-147.

Abdel-Kareem O (2010) Fungal deterioration of historical textiles and approaches for their control in Egypt. ePreservation Sci 7:40-47.

Abdel-Moneim NM, El-Ashry EH, Abdel-Azeem AM, Ali AN (2010) Marine-derived fungi isolated from invertebrates and seaweeds of Mediterranean coast at Alexandria as a source of bioactive secondary metabolites. In: The first international conference on basic and applied mycology, 9-11 March 2010, Assiut, Egypt, Abstract book. pp 55-56.

Abdel-Sater MA (1990) Studies on the mycoflora of the New Valley area, Western Desert, Egypt. Ph.D Thesis, Faculty of Science, Assiut University.

Abdel-Sater MA (2000) Soil fungi of the New Valley area, Western desert, Egypt. Bull Fac Sci Assiut Univ 29:255-271.

Abdel-Sater MA, A.H. M, Soliman ZSM (2016) Diversity of filamentous and yeast fungi in soil of citrus and grapevine plantations in the Assiut region, Egypt. Czech Mycol 68:183-214.

Abdel-Sater MA (1994) Cellulase activity and succession of fungi in soil amended with sodium chloride, organic matter and Ca-superphosphate. J Basic 
Microbiol 34:283-302.

https://doi.org/10.1002/jobm.3620340502

Abdul-Wahid O (1990) Fungal flora of cultivated soils and their role in the biological control of tomato Fusarium wilt in Ismailia governorate. Faculty of Science, Suez Canal University, Egypt

Abdullah SK, Aldossari MN, Al-Imara FJ (2010) Mycobiota of surface sediments in marshes of Southern Iraq. Marsh Bull 5:14-26.

Abo El-Lel A (1977) Studies on fungal population of the salt marshes of Egypt. Ph. D. Thesis, Faculty of Science, Mansoura University, Egypt.

Abou-Donia SA (2008) Origin, history and manufacturing process of Egyptian dairy products: An overview. Alexandria J Food Sci Technol 5:51-62.

Abu Deraz SS, Abdel-Azeem AM, Mansour SR (2016) Isolation and characterization of microbiota inhabiting Al-Aqsa mosque: Al-Quds, Palestine. LAP LAMBERT Academic Publishing. ISBN 978-3-65996786-3.

Ahmed AE, Al-kahtani MM, El-diasty EM, et al (2020) Diversity of toxigenic molds and mycotoxins isolated from dairy products : Antifungal activity of Egyptian marine algae on Aspergillus and Candida species. J Pure Appl Microbiol 14:215-232.

Al-Bedak O (2007) In: Catalouge of culture collection (2010). Assisut Univ Mycol Cent 45. https://doi.org/http://www.aun.edu.eg/aumc/pdf/AU

MC Catalog 2010.pdf

Al-Bedak O, Moubasher A (2020) Aspergillus gaarensis, a new addition to section Circumdati from soil of Lake El-Gaar in Wadi-El-Natron, Egypt. Stud Fungi 5:5965. https://doi.org/10.5943/sif/5/1/5

Ali M, AbdElfattah A, Khalil N, Sayed M (2017) Biological activities of the alkaloid Quinazoline extracted from Aspergillus nomius. Egypt $\mathrm{J}$ Bot $0: 565-582$.

https://doi.org/10.21608/ejbo.2017.1079.1096

Aljandali A (2016) Quantitative Analysis and IBM ${ }^{\circledR}$ SPSS $®$ Statistics. Statistics and Econometrics for Finance. https://doi.org/https://doi.org/10.1007/9783-319-45528-0.

Allam MG., Darwish AMG, Ayad EHE, et al (2017a) Lactococcus species for conventional Karish cheese conservation. LWT - Food Sci Technol 79:625-631. https://doi.org/doi: 10.3923/jm.2016.

Allam MG, Darwish AMG, Ayad EHE (2017b) Improve hygienic quality of Egyptian Karish cheese employing isolated antagonistic lactobacilli strains. Annu Res Rev Biol 19: https://doi.org/10.9734/ARRB/2017/37116

Ammar HAM, Ezzat SM, Houseny AM (2017) Improved production of kojic acid by mutagenesis of
Aspergillus flavus HAk1 and Aspergillus oryzae HAk2 and their potential antioxidant activity. 3 Biotech 7:. https://doi.org/10.1007/s13205-017-09054

Arabatzis M, Velegraki A (2013) Sexual reproduction in the opportunistic human pathogen Aspergillus terreus. Mycologia 105:71-79. https://doi.org/10.3852/11-426

Arenz BE, Blanchette RA, Farrell RL (2014) Fungal Diversity in Antarctic Soils. In: Cowan DA (ed) Antarctic Terrestrial Microbiology: Physical and Biological Properties of Antarctic Soils. Springer Berlin Heidelberg, Berlin, Heidelberg, pp 35-53.

Arya A, Shah A, Sadasivan S (2001) Indoor aeromycoflora of Baroda museum and deterioration of Egyptian mummy. Curr Sci 81:793-799.

Awaad AS, AL-Mudhayyif HA, Al-Othman MR, et al (2017) Amhezole, a novel fungal secondary metabolite from Aspergillus terreus for treatment of microbial mouth infection. Phyther Res 31:395-402. https://doi.org/10.1002/ptr.5760

Balbool BA, Abdel-Azeem AM, Kahlil WF, El-Kazzaz WM (2013) Bioprospecting as a conservation tool: the genus Aspergillus (Eurotium) in Egypt. In: Third International Congress on Fungal Conservation, Akyaka, Mugla, Turkey, Abstract book, p 36.

Balbool BA, Abdel-azeem AM (2020) Diversity of the culturable endophytic fungi producing L-asparaginase in arid Sinai, Egypt. Ital J Mycol 49:8-24. https://doi.org/10.6092/issn.2531-7342/10063

Barakat SM, El-leboudy AA, El-ansary MA (2019) Mycological evaluation of some dairy products served to student hostel with special reference to Aflatoxin M1 residue. Alexandria J Vet Sci 61:146-151. https://doi.org/10.5455/ajvs.32230

Bassuony NI, Abdel-Salam AF, Abdel-Ghany ZM, et al (2012) Chemical properties and microbiological quality for handling white cheese and effect of goat and camel milk on some pathogenic bacteria. J Food Dairy Sci, Mansoura Univ 3:647-667.

Bayman P, Baker JL, Doster MA, et al (2002) Ochratoxin production by the Aspergillus ochraceus group and Aspergillus alliaceus. Appl Environ Microbiol 68:2326-2329.

Besada WH, Yusef HM (1968) On the mycoflora of UAR soil. In: Proceedings of the Egyptian Academy of Sciences pp 103-109.

Billett H (1990) Hemoglobin and hematocrit. In: Clinical Methods: The history, physcial, and labratory examinations, 3rd editio. Butterworth Publishers, pp 718-719.

Blochwitz A (1929) Die Gattung Aspergillus. neue Spezies. Diagnosen. Synonyme. Ann Mycol 27:205-240.

Bonugli-Santos RC, Vasconcelos MR dos S, Passarini 
MRZ, et al (2015) Marine-derived fungi: Diversity of enzymes and biotechnological applications. Front Microbiol 6:1-15.

https://doi.org/10.3389/fmicb.2015.00269

Butinar L, Frisvad JC, N G-C (2011) Hypersaline waters- a potential source of foodborne toxigenic aspergilli and penicillia. FEMS Microbiol Ecol 77:186-199.

Chaudhuri H, Sachar GS (1934) A study of the fungus flora of the Punjab soils. Ann Mycol 32:90-100.

Conley CA, Ishkhanova G, McKay CP, Cullings K (2006) A preliminary survey of non-lichenized fungi cultured from the hyperarid Atacama Desert of Chile. Astrobiology 6:521-526.

Darwish S, El Hadidi N, Mansour M (2013) The effect of fungal decay on Ficus sycomorus wood. Int $\mathbf{J}$ Conserv Sci 4:271-282.

de Vries RP, Frisvad JC, van de Vondervoort PJI, et al (2005) Aspergillus vadensis, a new species of the group of black Aspergilli. Antonie van Leeuwenhoek, Int J Gen Mol Microbiol 87:195-203. https://doi.org/10.1007/s10482-004-3194-y

Delacroix EG (1897) Quelques espéces nouvelles. Bull la Société Mycol Fr 13:114-127.

Dörfelt H, Schmidt AR (2005) A fossil Aspergillus from Baltic amber. Mycol Res 109:956-960. https://doi.org/10.1017/S0953756205003497

El-Abyad M (1997) Biodiversity of fungal biota in Egypt. Up-dated check-list. [Publication Natl. Biodivers. Unit No. 7.] Egypt. Environ. Aff. Agency.

El-Abyad M, Abu-Taleb A (1993) II-Soil Fungi. Ecology of fungal flora. In: Publication of National Biodiversity Unit. No. 1. Habitat Diversity. Egyptian Environmental Affairs Agency, Egypt., pp 237-262.

El-Abyad M, Ismail I, Rizk M (1982) Ecological studies on the rhizospheres of some Egyptian halophilic plants. Egypt J Bot 25:91-107.

El-Abyad MS, Migahed FF (1989) Effects of treating seeds of Ricinus communis L. with some growth regulators on the rhizosphere microflora of developing plants. Egypt J Biol 32:173-190.

El-Badry S, Raslan A (2016) Mould contamination of some Egyptian cheese. Benha Vet Med J 30:28-33. https://doi.org/10.21608/bvmj.2016.31325

El-Diasty E., Salem R. (2007) Incidence of lipolytic and proteolytic fungi in some milk products and their public health significance. J Appl Sci Res 3:16841688.

El-Dohlob SM, Migahed FF (1985) Seed borne and rhizosphere fungi of four varieties of crop plants. In: 2nd Agricultural Conference of Botanical Science. pp 21-23.

El-fadaly HA, El-kadi SM, Hamad MN, Habib AA (2015a) Role of fungal enzymes in the biochemistry of
Egyptian Ras Cheese during Ripening Period. Open Access Libr J 2:e1819. https://doi.org/10.4236/oalib.1101819

El-fadaly HM, El-kadi SM, Hamad MN, Habib AA (2015b) Isolation and identification of Egyptian Ras Cheese ( Romy ) contaminating fungi during ripening period. J Microbiol Res 5:1-10. https://doi.org/10.5923/j.microbiology.20150501.01

El-Hissy FT, Moharram AM, El-Zayat SA (1990) Studies on the mycoflora of Aswan high Dam Lake, Egypt: Monthly variations. J Basic Microbiol 30:81-94.

El-Kady IA, Abdel-Hafez SII (1981) Production of sterigmatocystin by some species and varieties of Aspergillus nidulans group. Cryptogam Mycol 2:239244.

El-Kady IA, El-Maraghy MSS, Mostafa EM (1992) Contribution of the mesophilic fungi of different spices in Egypt. Mycopathologia 120:93-101.

El-kest MM, El-Hariri M, Khafaga NIM, Refai MK (2015) Studies on contamination of dairy products by Aflatoxin M1 and its control by probiotics. J Glob Biosci 4:1294-1312.

El-Kholy W, El-Khalek ABA, Mohamed SHS, et al (2016) Tallaga cheese as a new functional dairy product. Am J Food Technol 11:182-192. https://doi.org/10.3923/ajft.2016.182.192

El-Kholy WM, Soliman TN, Darwish AMG (2019) Evaluation of date palm pollen (Phoenix dactylifera $\mathrm{L}$.) encapsulation, impact on the nutritional and functional properties of fortified yoghurt. PLoS One 14:e0222789. https://doi.org/10.1371/journal.pone.0222789

El-Magraby OMO (1989) Contribution to the fungal flora and aflatoxin of straw in Egypt. Bull Fac Sci Assiut Univ 18:119-130.

El-Maraghy M (1989) The fungal flora and natural occurrence of mycotoxins of some leguminous varieties and hybrids seeds. Bull Fac Sci Assiut Univ 18:63-75.

El-Morsy E-SM (1999) Microfungi from the ectorhizosphere-rhizoplane zone of different halophytic plants from the Red Sea Coast of Egypt. Mycologia 91:228-236.

https://doi.org/10.1080/00275514.1999.12061012

El-Morsy EM (1990) Ecological studies on mangrove mycoflora. M. S. thesis, Faculty of Science, Mansoura University, Egypt.

El-Said AHM, Abdel-Hafez SII (1995) Seasonal variations of air borne fungi above banana fields in Qena, Upper Egypt. Cryptogam Mycol 16:101-109.

El-Zayat SA, Nassar M, El-Hissy F, et al (2008) Mycoflora associated with Hyoscyamus muticus growing under an extremely arid desert environment (Aswan region, Egypt). J Basic Microbiol 48:82-92.

ELbagory AM, Amal ME; Hammad AM; Salwa AD 
(2014) Prevalence of fungi in locally produced cheese and molecular characterization of isolated toxigenic molds. Benha Vet Med J 27:9-20.

Elramly MH, Leboudy AA, Al-Ansary MA (2019) Mycological evaluation of Egyptian Ras Cheese with special reference to mycotoxins. Alexandria J Vet Sci 63:33. https://doi.org/10.5455/ajvs.58688

Embaby EM, Awni NM, Abdel-galil MM, El-gendy HI (2015) Distribution of fungi and mycotoxins associated some foods. Middle East J Appl Sci 05:734-741.

Ezz-ElDin EK (1988) A Study of the soil fungal flora of the salt marsh ecosystem of North-Sinai. M. S. Thesis, Faculty of Science, Suez Canal University, Egypt.

Fennell DI, Raper KB (1955) New species and varieties of Aspergillus. Mycologia 47:68-89.

Findley K, Oh J, Yang J, et al (2013) Topographic diversity of fungal and bacterial communities in human skin. Nature 498:367-370. https://doi.org/10.1038/nature12171

Fox PF (1993) Cheese: Chemistry, physics and microbiology. Volume 2. Major cheese group, 2nd Editio. Springer Science+Business Media Dordrech.

Frisvad J, Thrane U (1987) Standardized high performance liquid chroma- tography of 182 mycotoxins and other fungal metabolites based on alkyl- phenone retention indices and UV-VIS spectra (diode array detection). J Chromatogr A 404:195-214.

Frisvad J, Thrane U (1993) Liquid chromatography of mycotoxins. In: Fujimoto H, Fujimaki (eds) Chromatography of mycotoxins: techniques and applications. Journal of Chro- matography Library 54 (Betina V, ed). Elsevier, Amsterdam, pp 253-372.

Frisvad JC, Hubka V, Ezekiel CN, et al (2019) Taxonomy of Aspergillus section Flavi and their production of aflatoxins, ochratoxins and other mycotoxins. Stud Mycol 93:1-63.

https://doi.org/10.1016/j.simyco.2018.06.001

Gams W, Christensen M, Onions AH, et al (1986) Infrageneric Taxa of Aspergillus. In: Samson RA, Pitt JI (eds) Advances in Penicillium and Aspergillus Systematics. Springer US, Boston, MA, pp 55-62.

Gautier M, Normand AC, Ranque S (2016) Previously unknown species of Aspergillus. Clin Microbiol Infect 22:662-669. https://doi.org/10.1016/j.cmi.2016.05.013

Hafez WA (2012) Comparative ecological studies on soil and rhizospheric fungi of maize and wheat plants in different areas in Minia Governorate Egypt. M.S. Thesis, Faculty of Science, Faculty of Science, ElMinia University, Egypt.

Hallen-Adams HE, Suhr MJ (2017) Fungi in the healthy 95 human gastrointestinal tract. Virulence 8:352-358. https://doi.org/10.1080/21505594.2016.1247140

Hamed SR (2016) Diversity of Aspergillus and Penicillium of farmland in Egypt. Int J Innov Stud Sci Eng Technol 2:2455-4863.

Hameed KGA (2016) Fungal diversity in different types of cheese and the effect of natamycin on their survival during Feta cheese manufacture and storage. J Adv Vet Anim Res 3:214-220.

Hammam GHA (2000) In: Catalouge of culture collection (2010). Assisut Univ Mycol Cent 45. https://doi.org/http://www.aun.edu.eg/aumc/pdf/AUMC \%20Catalog\%202010.pdf

Hassanin NI (1993) Detection of mycotoxigenic fungi and bacteria in processed cheese in Egypt. Int $\mathrm{Bi}$ odeterioration Biodegrad 31:15-23.

Hegazy TA, Abou Donia MA, Ibrahim MS, Hasballah AF (2014) Detection of aflatoxin M1 in milk and some dairy products in Damietta Governorate, Egypt. J Environ Sci 43:167-179.

Horn BW (2003) Ecology and population biology of aflatoxigenic fungi in soil. $\mathrm{J}$ Toxicol Toxin Rev 22:351-379.

Horn BW, Olarte RA, Peterson SW, Carbone I (2013) Sexual reproduction in Aspergillus tubingensis from section Nigri. Mycologia 105:1153-1163.

https://doi.org/10.3852/13-101

Horré R, Symoens F, Delhaes L, Bouchara JP (2010) Fungal respiratory infections in cystic fibrosis: a growing problem. Med Mycol 48:S1-S3.

https://doi.org/http://dx.doi.org/10.3109/13693786.2010 .529304

Houbraken J, De Vries R, Samson R (2014) Modern taxonomy of bio- technologically important Aspergillus and Penicillium species. Adv Appl Microbiol 86:199249.

Houbraken J, Samson R (2011) Phylogeny of Penicillium and the segregation of Trichocomaceae into three families. Stud Mycol 70:1-51.

Hubka V, Nováková A, Kolařík M, et al (2015) Revision of Aspergillus section Flavipedes: Seven new species and proposal of section Jani sect. nov. Mycologia 107:169208. https://doi.org/10.3852/14-059

Ibrahim ME (1999) Management of tomato fusarial wilt through integrated control. Ph.D thesis, Suez Canal University, Egypt.

IFP (2020) Index Fungorum Partnership (IFP), Accessed June 15, 2020. http//www.indexfungorum org/

IMI (2020) IMI Database, Accessed June 15, 2020. http://www.herbimi.info/herbimi/searchiminumber.htm

Ismail M, Abdel-Sater M, Zohri A (1995) Synoptic key to species of the Aspergillus nidulans-Emericella assemblage common to Egypt. Mycotaxon 53:391-405. 
Ismail MA, Abdel-Hafez SI, Moharram AM (2002) Aeromycobiota of Western Desert of Egypt. Asian J Sci Technol 3:1-9.

Jaime-Garcia R, Cotty P. (2010) Crop rotation and soil temperature influence the community structure of Aspergillus flavus in soil. Soil Biol Biochem 42:1842-1847.

Jaime-Garcia R, Cotty PJ (2006) Spatial relationships of soil texture and crop rotation to Aspergillus flavus community structure in South Texas. Phytopathology 96:599-607.

Jurjević Ž, Kubátová A, Kolařík M, V. H (2015) Taxonomy of Aspergillus section Petersonii sect. nov. encompassing indoor and soil-borne species with predominant tropical distribution. Plant Syst Evol 301:2441-62.

Khalifa MI, Al-ashmawy MA, Abdel-Khalik A, ElSherbini M (2013) Mycological evaluation of serving some dairy products with special reference to mycotoxins production in Azhar University student hostels. World J Dairy Food Sci 8:165-170. https://doi.org/10.5829/idosi.wjdfs.2013.8.2.76133

Khalifa MI, Shata RR (2018) Mycobiota and aflatoxins B1 and M1 levels in commercial and homemade dairy desserts in Aswan City, Egypt. J Adv Vet Res 8:4348.

Khalil AMA, El-sheikh HH, Sultan MH (2013) Distribution of fungi in mangrove soil of coastal areas at Nabq and Ras Mohammed Protectorates. Int J Curr Microbiol Appl Sci 2:264-274.

Klich MA (2002) Biogeography of Aspergillus species in soil and litter. Mycologia 94:21-27.

Klitgaard A, Iversen A, Andersen, MR et al. (2014) Aggressive dereplication using UHPLC-DAD-QTOF - screening extracts for up to 3000 fungal secondary metabolites. Anal Bioanal Chem 406:1933-1943.

Kocsubé S, Perrone G, Magistà D, et al (2016) Aspergillus is monophyletic: evidence from multiple gene phylogenies and extrolites profiles. Stud Mycol 85:199-213.

Kowalik R, Sadurska I (1973) Microflora of papyrus from samples of Cairo Museum. Stud Conserv 18:1-24.

Krug J, Khan R (1999) Soil fungi from eastern Dakhleh Oasis. In: Churcher C, Mills A (eds) Reports from the Survey of Dakhleh Oasis, Western Desert of Egypt, 1977-1987. Dakhleh Oasis Project Monograph 2: Oxbow Monograph. Oxbow Books, Oxford, U.K., pp 69-71.

Levic J, Gosic-Dondo S, Ivanovic D, et al (2013) An outbreak of Aspergillus species in response to environmental conditions in Serbia. Pestic i fitomedicina 28:167-179. https://doi.org/10.2298/pif13031671
Mahmoudi SAZ, Abou El-Fadl M, Elmofty MK (1964) Studied on the rhizosphere microflora of a desert plants. Folia Microbiol (Praha) 9:1-8.

Marchal, Marchal ÉJ (1893) Revue mycol. Toulouse 15:101

Marguet C, Favennec L, Matray O, et al (2012) Clinical and microbiological efficacy of micafungin on Geosmithia argillacea infection in a cystic fibrosis patient. Med Mycol Case Rep 1:79-81. https://doi.org/10.1016/j.mmcr.2012.08.004

Marín S, Ramos AJ, Sanchis V (2012) Modelling Aspergillus flavus growth and aflatoxins production in pistachio nuts. Food Microbiol 32:378-388. https://doi.org/10.1016/j.fm.2012.07.018

Mazen MB, Abdel-Hafez SII, Shaban GMM (1984) Survey on the mycoflora of Egyptian wheat grains and their lemmae and paleae. Mycopathologia 85:155-159. https://doi.org/10.1007/BF00440945

McSweeney PLH (2007) Cheese problems solved. CRC Press LLC. Woodhead Publishing Limited, Abington Hall, Abington, Cambridge CB21 6AH, England

Melchers L (1931) A check list of plant diseases and fungi occurring in Egypt. Trans Kansas Acad Sci 34:41-106.

Moharram AM, Abdel-Gawad KM, Megalla SE, Mahmoud ALE (1989a) Fungal flora of poultry feedstuff ingredients. J Basic Microbiol 29:29 (8), 491-499.

Moharram AM, Abdel- Mallek AY, Abdel- Hafez AII (1989b) Mycoflora of anise and fennel seeds in Egypt. J Basic Microbiol 29:427-435. https://doi.org/10.1002/jobm.3620290709

Moharram AM, Ahmed HE, Nasr SA (2013) Otomycosis in Assiut, Egypt. J Basic Appl Mycol 4:1-11.

Moharram AM, Haleem AAA El, Refaie RRS (2018) Mycological and mycotoxins analysis of Kareish and soft cheese in Assiut, Egypt. Am J Microbiol Res 6:165-172. https://doi.org/10.12691/ajmr-6-4-4

Montasir AH, Mostafa MA, Elwan SH (1956a) Development of soil microflora under Zygophyllum album L. and Zygophyllum coccineum L. Ain Shams Sci Bull 1:9-22.

Montasir AH, Mostafa MA, Elwan SH (1956b) Development of soil microflora in relation to vegetation along a transect line at yellow hills, North Cairo. Ain Shams Sci Bull 1:23-32.

Moubasher A-AH, Abdel-Sater MA, Soliman Z (2018a) Biodiversity of filamentous and yeast fungi in citrus and grape fruits and juices in Assiut area, Egypt. J Microbiol Biotechnol Food Sci 7:353-365. https://doi.org/10.15414/jmbfs.2018.7.4.353-365

Moubasher A (1993) Soil Fungi of Qatar and Other Arab Countries. Sci Appl Res Centre, Univ Qatar.

Moubasher A, Abdel-Hafez S (1978a) Studies on the mycoflora of Egyptian soils. Mycopathologia 63:3-10.

Moubasher A, Abdel-Sater M, Soliman Z (2013) Contribution to the mycobiota of Egypt Emericella 
stella-maris Zalar, Frisvad \& Samson 2008, a new record to Egypt. J Basic Appl Mycol 4:85-87.

Moubasher A, Abdel-Sater M, Soliman Z (2018b) First records of Aspergillus porphyreostipitatus and Aspergillus carlsbadensis since their original descriptions. Czech Mycol 70:70(1): 67-82. https://doi.org/ISSN 1805-1421

Moubasher A, Moharram AM, Ismail MA (2011) The genus Aspergillus and its teleomorphs. Part 1. AUMC Descr 1-35.

Moubasher AAH, Abdel-Sater MA, Soliman ZSM (2018c) Yeasts and filamentous fungi associated with some dairy products in Egypt. J Mycol Med 28:76-86. https://doi.org/10.1016/j.mycmed.2017.12.003

Moubasher AAH, Abdel-Sater MA, Soliman ZSM (2018d) Diversity of floricolous yeasts and filamentous fungi of some ornamental and edible fruit plants in Assiut area, Egypt. Curr Res Environ Appl Mycol 8:135161. https://doi.org/10.5943/cream/8//12

Moubasher AH, Abdel-Hafez SI (1978b) Study on the mycoflora of Egyptian soils. Mycopathologia 63:310.

https://doi.org/https://doi.org/10.1007/BF00473153

Moubasher AH, Abdel-Hafez SII, Bagy MMK, AbdelSatar MA (1990a) Halophilic and halotolerant fungi in cultivated desert and salt marsh soils from Egypt. Acta Mycol XXVI:65-81.

Moubasher AH, Abdel-Hafez SII, EI-Maghraby OMO (1985) Studies on soil mycoflora of Wadi Bir-EI-Ain, Eastern desert, Egypt. Cryptogam Mycol 6:129-143.

Moubasher AH, Abdel-Hafez SII, El-Maghraby OMO (1988) Seasonal fluctuations of soil and air borne fungi of Wadi Bir- El-Ain in Eastern Desert of Egypt. Nat Monspel Ser Bot 52:57-70.

Moubasher AH, Abdel-Kader MIA, El-Kady IA (1978) Toxigenic fungi isolated from Roquefort cheese. Mycopathologia 66:187-190.

Moubasher AH, Abdel-Sater MA, Soliman Z (2016) Biodiversity and molecular characterization of yeast and filamentous fungi in the air of citrus and grapevine plantations in Assiut area, Egypt. Mycosphere 7:236-261.

https://doi.org/10.5943/mycosphere/7/3/1

Moubasher AH, El-Dohlob SM (1970) Seasonal fluctuations of Egyptian soil fungi. Trans Br Mycol Soc 54:45-51. https://doi.org/10.1016/s0007-1536(70)80122-x

Moubasher AH, El-Sharouny HMM, Badran RA (1990b) Fungi associated with cellulose exposed to Nile water refuses of Qena city in Upper Egypt. Egypt $\mathrm{J}$ Bot 33:1-14.

Moubasher AH, Elnaghy MA, Abdel-Hafez SI (1972) Studies on the fungus flora of three grains in Egypt.
Mycopathol. Mycol. Appl. 47:261-274.

Moubasher AH, Ismail MA, Hussein NA, Gouda HA (2015) Osmophilic/osmotolerant and halophilic/halotolerant mycobiota of soil of Wadi El-Natrun regions. J Basic Appl Mycol 6:27-42.

Moubasher AH, Moharram AM, Ismail MA (2010) Catalogue of culture collection (AUMC).

Moubasher AH, Moustafa AF (1974) Air-borne fungi at Assiut, Egypt. Egypt J Bot 17 (2,3): 135-149.

Moubasher AH, Moustafa AF (1970) A survey of Egyptian soil fungi with special reference to Aspergillus, Penicillium and Penicillium-related genera. Trans $\mathrm{Br}$ Mycol Soc 54:35-44. https://doi.org/10.1016/s00071536(70)80121-8.

Moubasher AH, Moustafa AF (1972) Aspergillus aegyptiacus sp. nov. Egypt. Egypt J Bot 15:153-154.

Moubasher AH, Soliman Z (2011) Aspergillus assiutensis , a new species from the air of grapevine plantation , Egypt. J Basic Appl Mycol 2 2:83-90.

Mouchacca J (1971) Pseudeurotium desertorum sp. nov. Rev Mycol 36:123-127.

Mouchacca J (1973a) Les Thielavia des sols arides: Espèces nouvelles et analyse générique. Bull Trimest la Société Mycol Fr 89:295-311.

Mouchacca J (1977) Sur un nouveau Discomycetes Ascobolus egyptiacus Travaux dédiès à G. ViennotBourgin. Société Fr Phytopathol Paris 236-267.

Mouchacca J (1985) Les champignons. In: Balout L, Desroches-Noblecourt C, Roubet C (eds) La Momie de Ramsès II : Contribution scientifique à l'égyptologie. Editions Recherche sur les Civilisations, Paris, p 427.

Mouchacca J (1995) Check-list of novel fungi from the Middle East described mainly from soil since 1930. Sydowia 47:240-257.

Mouchacca J (1999) A list of novel fungi described from the Middle East, mostly from non-soil substrata. Nov Hedwigia 68:149-174.

Mouchacca J (2005) Mycobiota of the arid Middle East: check-list of novel fungal taxa introduced from 1940 to 2000 and major recent biodiversity. J Arid Environ 60:359-387.

Mouchacca J (1982) Étude analytique de la mycoflore de quelques sols de régions arides de l'Égypte. Ph.D thesis, Université Pierre et Marie Curie: Muséum national d'histoire naturelle.

Mouchacca J (1973b) Deux Alternatria des sols arides d'Égypte: A. Chlamydosporum sp. nov. et $A$. Phragmospora Van Emden. Mycopathol Mycol Appl 50:217-225. https://doi.org/10.1007/BF02053370

Mouchacca J, Joly P (1974) Etude de la mycoflore des sols arides de l'Egypte. I. Le genre Penicillium. Rev d'Ecologie Biol du Sol 11:67-88.

Mouchacca J, Joly P (1976) Etude de la mycoflore des sols 
arides de l'Egypte. II. Le genre Aspergillus. Rev d'Ecologie Biol du Sol 13:293-313.

Mouchacca J, Nicot J (1973) Les Fusariella des sols arides. Rev Mycol 37:168-182.

Moustafa A, Abdel-Azeem A (2011) An annotated checklist of Ascomycota reported from soil and other terricolous substrates in Egypt. J Basic Appl Mycol 2:1-27.

Moustafa AF (1975) Osmophilous fungi in the salt marshes of Kuwait. Can J Microbiol 21:1573-1580.

Mustafa A, Abdel-Azeem A, Salem F (2013) Surveying and exploitation of some taxa for extracellular biosynthesis of silver nanoparticles. In: Third International Congress on Fungal Conservation, Akyaka, Mugla, Turkey, 11-15 November 2013. p 44

Muthomi JW, Mureithi BK, Chemining'wa, G.N. Gathumbi JK, Mutitu EW (2012) Aspergillus species and Aflatoxin B1 in soil, maize grain and flour samples from semi-arid and humid regions of Kenya. Int J AgriScience 2:22-34.

Naguib AI, Mouchacca J (1970) The mycoflora of Egyptian desert soils. Bull l'Institut d'Egypte 52:3761.

Nassar MSM (1998) Soil mycoflora of Wadi AbuSubayrah at Aswan region at Eastern Desert of Egypt. Egypt J Bot 38:21-46.

Natrass R (1932) Preliminary notes on some entomogenous fungi in Egypt. Tech Ser Bull Minist Agric Egypt 120:1-9.

Okuda T (1994) Variation in colony characteristics of Penicillium strains resulting from minor variations in culture conditions. Mycologia 86:259-262.

Okuda T, Klich M, Seifert K, Ando K (2000) Media and incubation effect on morphological characteristics of Penicillium and Aspergillus. In: Samson R, Pitt J (eds) Penicillium and Aspergillus; Integration of modern taxonomic methods for Penicillium and Aspergillus classification.

Paulson D (2008) Nonparametric statistical methods. In: Paulson D (ed) Biostatistics and microbiology: A survival manual. pp 121-162.

doi: 10.1007/978-0-387-77282-0_7

Peterson S (2008) Phylogenetic analyses of Aspergillus species using DNA sequences from four loci. Mycologia 100:205-226.

Peterson S (2000) Phylogenetic relationships in Aspergillus based on rDNA sequence analysis. In: Samson R, Pitt $\mathrm{J}$ (eds) Integration of modern taxonomic methods for Penicillium and Aspergillus classification. Harwood Academic Publishers, Amsterdam, pp 323-355.

Peterson S, Varga J, Frisvad J, Samson R (2008) Phylogeny and subgeneric taxonomy of Aspergillus. In: Varga, J, Samson R (ed) Aspergillus in the genomic era. Wageningen: Wageningen Academic Publishers, pp 33-56.

Ragab M (1956) A contribution to the fungi of Egypt. Mycologia 48:167-168.

Raper K, Fennell D (1965) The Genus Aspergillus. Williams and Wilkins, Baltimore

Reichert I (1921) Die Pilzflora Äegypten. Engler's Bot Jahrbüchen 56:595-727.

Sabet Y (1939) On some fungi isolated from soil in Egypt. Bull Fac Sci Fouad I Univ Egypt Univ Cairo 19:1-112.

Sabet Y (1935) A preliminary study of Egyptian soil fungi. Bull Fac Sci Egypt Univ Cairo 5:1-29.

Salama AM, Elbatanoni K, Ali MI (1971) Studies on the fungal flora of Egyptian soils. I. Western Mediterranean coast and Libyan Desert. United Arab Repub J Bot 14:99-114.

Salem FM, Abdel-Azeem AM (2014) Screening of anticancer metabolites produced by endophytic fungi: Of Some Medicinal Plants in Saint Katherine Protectorate. LAP LAMBERT Academic Publishing GmbH KG, ISBN 978-3-659-53697-7.

Samson RA, Mouchacca J (1975) Additional notes on species of Aspergillus, Eurotium and Emericella from Egyptian desert soil. Antonie Van Leeuwenhoek 41:343-351. https://doi.org/10.1007/BF02565069

Samson RA, Mouchacca J (1974) Some interesting species of Emericella and Aspergillus from Egyptian desert soil. Antonie Van Leeuwenhoek 40:121-131. https://doi.org/10.1007/BF00394559

Samson RA, Varga J (2010) Molecular systematics of Aspergillus and its teleomorphs. In: Machida G (ed) Aspergillus: molecular biology and genomics. Horizon Press, pp 19-40

Samson RA, Varga J, Witiak SM, Geiser DM (2007) The species concept in Aspergillus: Recommendations of an international panel. Stud Mycol 59:71-73. https://doi.org/10.3114/sim.2007.59.09

Samson RA, Visagie CM, Houbraken J, et al (2014) Phylogeny, identification and nomenclature of the genus Aspergillus. Stud Mycol 78:141-173. https://doi.org/10.1016/j.simyco.2014.07.004

Seddek NH, Gomah NH, Osman DM (2016) Fungal flora contaminating Egyptian Ras cheese with reference to their toxins and enzymes. Food Sci Technol 4:64-68. https://doi.org/10.13189/fst.2016.040403

Shaaban M, Nasr H, Hassan AZ, Asker MS (2013) Bioactive secondary metabolites from endophytic Aspergillus fumigatus: Structural elucidation and bioactivity studies. Rev Latinoam Quim 41:50-60.

Shindia AAE (1990) Studies on fungal degradation of composts in Egypt. Ph.D thesis, Faculty of Science, Zagazig University. 
Sivakumar T, Ravikumar M, Sivakumar N (2006) Abundance of mangrove fungi along the east coast of Tamil Nadu, India. Asian J Microbiol Biotechnol Environ Sci 18:589-594.

Sklenář F, Jurjević Ž, Zalar P, et al (2017) Phylogeny of xerophilic aspergilli (subgenus Aspergillus) and taxonomic revision of section Restricti. Stud Mycol 161-236.

Smedsgaard J (1997) Micro-scale extraction procedure for standardized screening of fungal metabolite production in cultures. J Chromatogr A 760:264-270.

Soliman E (2016) A taxonomic analysis of fungi collected and described from Egypt up to 1931. Stud Fungi 1:11-33. https://doi.org/10.5943/sif/1/1/2

Stolk AC (1964) Aspergillus flaschentraegeri sp.nov. Trans Br Mycol Soc 47:123-126. https://doi.org/10.1016/s0007-1536(64)80087-5

Subhan M, Faryal R, Macreadie I (2016) Exploitation of Aspergillus terreus for the production of natural statins. J Fungi 2:. https://doi.org/10.3390/jof2020013

Subramanian CV (1972) The perfect states of Aspergillus. Curr Sci 41:755-761.

Tang Y, Lian B, Dong H, et al (2012) Endolithic bacterial communities in dolomite and limestone rocks from the Nanjiang Canyon in Guizhou Karst area (China). Geomicrobiol J 29:213-225.

Thom C, Church M (1926) The Aspergilli. Williams \& Wilkins, Baltimore, MD.

Thom C, Church MB (1921) Aspergillus flavus, A. oryzae, and associated species. Am J Bot 8:103-126.

Thom C, Raper K (1945) A Manual of the Aspergilli. Williams \& Wilkins, Baltimore, MD.

Thomas GM, Poinar GO (1988) A fossil Aspergillus from Eocene Dominican amber. J Paleontol 62:141-143. https://doi.org/10.1017/S0022336000058996

Tripathi M, Joshi Y (2015) Endolichenic fungi in Kumaun Himalaya: A case study. In: Upreti DK, Divakar PK, Shukla V, Bajpai R (eds) Recent Advances in Lichenology: Modern methods and approaches in lichen systematics and culture techniques, Volume 2. Springer India, New Delhi, pp 111-120.

Tsang CC, Tang JYM, Lau SKP, Woo PCY (2018) Taxonomy and evolution of Aspergillus, Penicillium and Talaromyces in the omics era - Past, present and future. Comput Struct Biotechnol J 16:197-210. https://doi.org/10.1016/j.csbj.2018.05.003

Varga J, Frisvad JC, Samson RA (2010) Aspergillus sect. Aenei sect, nov., a new section of the genus for $A$. karnatakaensis sp. nov. and some allied fungi. IMA Fungus 1:197-205.

Varga J, Kevei F, Hamari Z, et al (2000) Genotypic and phenotypic variability among black aspergilli. In: Samson R, Pitt J (eds) Integration of modern taxonomic methods for Penicillium and Aspergillus classification. Amsterdam: Harwood Academic Publishers, pp 397411.

Visagie C, Hirooka Y, Tanney, JB et al. (2014) Aspergillus, Penicillium and Talaromyces isolated from in house dust samples collected around the world. Stud Mycol 78:63-139.

Yassein AS, El- Said AHM, El- Dawy EGA (2020) Biocontrol of toxigenic Aspergillus strains isolated from baby foods by essential oils. Flavour Fragr J 35:182-189. https://doi.org/10.1002/ffj.3551

Younis G, Ibrahim D, Awad A, ELBardisy MM (2016) Determination of Aflatoxin M1 and Ochratoxin A in milk and dairy products in supermarkets located in Mansoura City, Egypt. Adv Anim Vet Sci 4:114-121. https://doi.org/http://dx.doi.org/10.14737/journal.aavs/2 016/4.2.114.121

Yu Z, Zhang B, Sun W, et al (2013) Phylogenetically diverse endozoic fungi in the South China Sea sponges and their potential in synthesizing bioactive natural products suggested by PKS gene and cytotoxic activity analysis. Fungal Divers 58:127-141.

https://doi.org/10.1007/s13225-012-0192-7

Zidan Y, Handoussa T, Hosni H, El Hadidi N (2006) The conservation of a wooden Graeco- Roman coffin box, e-Preservation. Science (80- ) 3:27-33.

Zohri AA, Saber S, Youssef M, Abdel-Kareem M. (2014) Isolation and identification of toxigenic fungi from foodstuffs at Sohag Governorate, Egypt. J Environ Stud 13:1-12.

Zommara MA, Rashed MA (2004) Moulds and yeasts incidence in samples from ras cheese surface and in their production areas air. In: The 4th Scientific Conference of Agricultural Sciences, Assiut, Egypt. pp 311-321. 